Working Paper 95-12

Economics Series 08

March 1995
Departamento de Economía

Universidad Carlos III de Madrid

Calle Madrid, 126

28903 Getafe (Madrid)

Fax (34 1) 624-9875

\title{
INCOMPLETE MARKETS IN INFINITE HORIZON: DEBT CONSTRAINTS VERSUS NODE PRICES*
}

\author{
Monique Florenzano and Pascal Gourdel*
}

\begin{abstract}
The general equilibrium model with incomplete markets is here extended to infinite horizon economies populated by a finite number of infinitely-lived agents. The crucial issue which divides the infinite horizon setting from the finite horizon setting is in the nature of borrowing constaints which added to spot constraints define a plausible budget set for individual agents. The paper relates seven alternative definitions of equilibrium and states corresponding equilibrium existence theorems when assets are one-period and purely financial.
\end{abstract}

Key words:

Stochastic equilibrium; Incomplete markets; Infinite horizon; Purely financial, One-period securities; No-arbitrage asset pricing.

"Florenzano, CNRS-CEPREMAP; Gourdel, CERMSEM, Université de Paris I. A preliminary version of this paper was first presented at the Conference on Current Trends in Economics: Theory and Application at Cephalonia in May 1993. A more substantial version (Couverture orange Cepremap no. 9329), with part of our equivalence results, was presented at the Workshop on General Equilibrium at Alicante in December 1993. We are grateful to David Cass, Mario Páscoa, Manuel Santos and Bill Zame for stimulating conversations and to Michael Magill and Martine Quinzii for access to the successive versions of their work. We would also like to thank the Universidad Carlos III de Madrid for its hospitality while part of this work was completed. 


\section{Introduction}

This paper deals with the equilibrium existence in infinite horizon economies with a finite number of agents and incomplete markets. It is in the line of a recent literature beginning with Levine (1989) and going mainly through the successive versions of papers by Hernandez and Santos (1991), Magill and Quinzii [(1994), first circulated in 1992], Levine and Zame (1992), Santos and Woodford [(1993), previous version in 1992], Hernandez and Santos (1994).

As is now very well known, in an incomplete market model that extends over an infinite time horizon, the first problem to solve is to define the individual budget sets so as to rule out Ponzi schemes while preserving incompleteness of markets. Several suggestions have been made that provide not less than seven alternative definitions of equilibrium. The different definitions share the property of generalizing the usual one for finite horizon economies with incomplete markets. The crucial difference between them is in the condition which, added to the spot budget constraints, prevents the agents from rolling over indefinitely their debts.

A first type of conditions limits explicitely or implicitely the possible amount of debt hold in each date-event at the beginning of the period (debt constraints) or at the end of the period (borrowing limits). Such explicit restrictions may be personalized. Then, as asset prices, commodity prices, consumption plans and portfolio plans, the systems of debt constraints or borrowing limits are components of the equilibrium. But the explicit restrictions may also be endogenous, in the spirit of what results from an institutional debt regulation. This is the case for the finitely effective debt constraints that prescribe in each node an almost finite-time debt-repayment.

A second type of conditions is more controversial. In a kind of solvency requirement, adapted from Hernandez and Santos (1991), the agents can be restricted not to hold debt in excess of the present value of their future savings or alternatively the agents cannot hold debt in the limit. Present values are computed with respect to node prices that are not uniquely determined in the case of incomplete markets and can differ among agents. This leads to a notion of equilibrium with present value node prices where the node prices are components of the equilibrium. An alternative condition, due to Magill and Quinzii (1994), requires an asymptotic evolution of the present value of net indebtedness of the agents in each subtree of the event-tree defining the stochastic structure of the model. This condition, in the spirit of the subgame perfection, leads to the MQ-equilibrium defined in this paper; it also uses personalized node prices. 
As we will see later, if the individual present value price is summable, each budget set with node prices coincides with one budget set with debt constraints. However, in general, the budget sets involved in different equilibrium definitions do not coincide. We will state in this paper several inclusions, some of them are strict. Hernandez and Santos (1994) establish that a budget set with debt constraints which is of interest, the finitely effective budget set, is the intersection of all budget sets relative to some admissible system of node prices and also the intersection of all associated Arrow-Debreu budget sets. In view of inclusions which will be proved in this paper, the finitely effective budget set coincides also with the intersection of all M-Q budget sets and with the intersection of all budget sets with loose and consistent debt constraints. But, generally, it strictly contains the budget sets associated with bounded debt constraints or with implicit borrowing limits.

Our first result is an equilibrium existence result for what appears to be a weak equilibrium concept : an equilibrium with household specific loose and consistent debt constraints. Actually, as Hernandez and Santos (1991), we restrict ourselves to the case of nominal assets that expire after one period. In this context, we generalize a result that is well known for finite horizon economies with purely nominal assets and prove that any asset price process that prevents arbitrage can be embedded in an equilibrium of the infinite horizon economy. Obviously, the existence of equilibrium for the case of oneperiod numeraire assets follows. Classically, in the proof of this result, we construct our equilibrium as the limit for convenient topologies of each component of some particular equilibrium of the truncated economies which are finite horizon economies with incomplete markets.

Then, comparing the different approaches, our main result is the equivalence, under appropriate assumptions, of six apparently quite distinct notions of equilibrium which give in fact the same equilibrium allocations and prices. Added to the first theorem, the equivalence theorem establishes equilibrium existence results comparable with the ones of Magill and Quinzii (1994), Levine and Zame (1992), Hernandez and Santos (1994) for the case of one-period numeraire assets. Its main interest seems to lie in the fact that it justifies the controversial node price approach. Indeed, at equilibrium, as proved by Hernandez and Santos (1994), the agents are constrained by all admissible systems of node prices but each agent uses a particular system of node prices to solve its optimization problem. With incomplete markets, without more assumptions, the particular systems of node prices may not coincide. Under special assumptions, Hernandez and Santos (1991) stated that the agents may agree about the choice of any same system of node prices.

The paper is organized as follows. In the next section, we present more precisely 
the model and we discuss different notions of equilibrium. In section 3 , we state our existence result of equilibrium with debt constraints. In section 4 , we compare the different definitions of equilibrium. Finally, some proofs are postponed in an appendix.

\section{The model and the definitions of equilibrium}

\subsection{The model}

We consider an exchange economy with countably many periods $t \in\{0,1, \ldots\}$, and a finite number $m$ of infinite-lived agents. The stochastic structure of the model is described by an infinite event-tree $S$, with a unique initial node denoted by 0 at date 0 and a finite branching number at each node of the tree. For every $t, S_{t}$ denotes the set of nodes occuring at date $t$ and $S^{t}$ the set of nodes occuring before or during time period $t$. For any node $s \in S_{t}, s^{+}$denotes the (finite) set of immediate successors of $s$ at date $(t+1)$; the number of elements of $s^{+}$is denoted by $b(s)$ and is called the branching number of the event-tree $S$ at node $s$. If $s \in S_{t}, t>0, s^{-}$denotes the (unique) node that immediately precedes $s$ at date $(t-1)$. We will also use the notation $S^{+}(s)$ for the set of successors of $s$ and $S(s)$ will denote the subtree starting at s. In particular, we have $S(s)=S^{+}(s) \cup\{s\}$ and $S=S(0)$.

At every node in the event-tree $S$ described above, a finite number $L$ of commodities are available and consumers trade commodities on spot markets. As in Bewley (1972), we take the commodity space to be $\ell_{\infty}(S \times L)$. Each consumer is classically described by a consumption set $X^{i} \subset \ell_{\infty}(S \times L)$, a preference relation $\checkmark^{i}$ on $X^{i}$ and a state-dependent initial endowment $\omega^{i} \in X^{i}$. On the other hand, at every node $s$, agents participate in a financial market. We assume that the set $J_{s}$ of financial instruments available at $s$ is finite and that the only available financial assets are purely financial, one-period securities. Let $r^{j}(\sigma)$ be the return in units of account that asset $j \in J_{s}$ promises to pay at $\sigma \in s^{+}$and $r(\sigma)=\left(r^{j}(\sigma)\right)_{j \in J_{s}}$. The matrix of returns $\left(b(s)\right.$ rows, $J_{s}$ columns) $R_{s}=\left(r^{j}(\sigma)\right)_{\sigma \in s^{+}, j \in J_{s}}$ describes the financial structure at $s$ and $R=\prod_{s \in S} R_{s}$ denotes the financial structure of the economy. Finally, the economy is summarized by the list of data

$$
\mathcal{E}=\left(S, R,\left(X^{i}, \succsim^{i}, \omega^{i}\right)_{i=1}^{m}\right)
$$

We will make on $\mathcal{E}$ the following assumptions:

For each agent $i$,

$C 1-\quad X^{i}=\ell_{\infty}^{+}(S \times L)$ 
C2 - $\omega^{i} \in \operatorname{int}\left(X^{i}\right)$.

C3 - $\succsim^{i}$ is a complete, convex, monotone, Mackey continuous preorder, such that for every $\lambda \in\left[0,1\left[\right.\right.$ and every $x^{i i} \succ^{i} x^{i}$ one has $\lambda x^{i}+(1-\lambda) x^{i} \succ^{i} x^{i}$. The preorder is monotone in the sense that for each $x^{i} \in X^{i}$ and for each $y \in \ell_{\infty}^{+}(S \times L)$, one has $x^{i}+y \succsim^{i} x^{i}$.

Convexity and Mackey continuity mean that for all $\bar{x}^{i} \in X^{i}$, the set $\left\{x^{i} \in X^{i} \mid x^{i} \succsim^{i} \bar{x}^{i}\right\}$ is convex and closed in the Mackey topology $\tau\left(\ell_{\infty}(S \times L), \ell_{1}(S \times L)\right)$ (hence closed in the weak topology $\left.\sigma\left(\ell_{\infty}(S \times L), \ell_{1}(S \times L)\right)\right)$ and the set $\left\{x^{i} \in X^{i} \mid x^{i} \succ^{i} \bar{x}^{i}\right\}$ is open in the same Mackey topology.

C4- For all $s, 1_{s}$ is extremely desirable for agent $i$ in the sense that for each $x^{i} \in X^{i}$ and for each node $s$, one has $x^{i}+1_{s} \succ^{i} x^{i}$. Obviously, $1_{s}$ is the direction defined by $1_{s}\left(s^{\prime}, \ell\right)=0$ if $s^{\prime} \neq s, 1_{s}(s, \ell)=1$, for all $\ell=1, \ldots, L$.

Before introducing the next assumption, we will give some notations. We will denote by $F$, the set $\left\{x^{i} \in \ell_{\infty}^{+}(S \times L)\|\| x^{i}\left\|_{\infty} \leq 2\right\| \sum_{i=1}^{m} \omega^{i} \|_{\infty}\right\}$. We emphasize that this set contains all feasible allocations. If $D \subset S$, we will also use the notation $\chi_{D}$ for the characteristic function of $D$; so if $a^{i}$ is a process on $S$, the element $a^{i} \chi_{D}$ is defined by

$$
\left(a^{i} \chi_{D}\right)(s)= \begin{cases}a^{i}(s) & \text { si } s \in D \\ 0 & \text { si } s \notin D .\end{cases}
$$

This notation will be used for both the consumption and the portfolio plans.

C5 - There exists $\beta \in[0,1[$ such that for each agent $i$, for every node $s$ and for all $x^{i} \in F$,

$x^{i} \chi_{S \backslash S+(s)}+1_{s}+\beta x^{i} \chi_{S+(s)} \succ^{i} x^{i}$.

We will also assume that the financial markets verify:

$F$. In each node $s$, there exists a non-risky asset $j \in J_{s}$ (i.e. $r^{j}(\sigma)>0$ for each node $\sigma \in s^{+}$). There is no restriction on the space of portfolios. At node $s$, it is equal to the whole space $\mathbb{R}^{J}$.

On the consumption side, our assumptions are standard. As Bewley (1972), Magill and Quinzii (1994), Levine and Zame (1992), we restrict the consumers to positive bounded consumptions plans. Assumption $C 2$ implies the assumption of commensurability made by Levine and Zame (1992). Together with $C 4$, Assumption $C 3$ implies that for each $x^{i} \in X^{i}$ and for each $y \in \ell_{\infty}^{+}(S \times L)$ satisfying $y(s, \ell) \neq 0$ for some node $s$ and every commodity 
$\ell$, one has $x^{i}+y \succ^{i} x^{i}$. This assumption of monotonicity is weaker than the strong monotonicity : for each $x^{i} \in X^{i}$ and for each $y \in \ell_{\infty}^{+}(S \times L) \backslash\{0\}$, one has $x^{i}+y \succ^{i} x^{i}$. Continuity in the Mackey topology $\tau\left(\ell_{\infty}(S \times L), \ell_{1}(S \times L)\right)$ is the natural extension of the similar assumption made by Bewley (1972) to the case of an economy with time and uncertainty; it expresses the idea of the impatience of the agents. For a discussion of this assumption, see Bewley (1972), Brown and Lewis (1981), Mas-Colell and Zame (1991).

The assumption $C 5$ is a condition of uniform impatience of the agents with respect to future consumption at each node. Similar assumptions can be found in Santos and Woodford (1993), Levine and Zame (1992), Hernandez and Santos (1994). C5 is satisfied if the preferences of agent $i$ are represented by an additively separable utility function :

$$
u^{i}\left(x^{i}\right)=\sum_{s \in S} \rho(s) \delta_{i}^{t(s)} v^{i}\left(x^{i}(s)\right)
$$

where $\rho$ defines a probability on each $\left.S_{T}, \delta_{i} \in\right] 0,1\left[\right.$ is a discount factor and $v^{i}$ is a continuous, increasing concave function on $\mathbb{R}_{+}^{L}$ satisfying $v^{i}(0)=0$. The assumption $C 5$ reinforces the assumptions $C 3$ and $C 4$. Indeed, one has the following lemma :

Lemma 1. [Magill and Quinzii (1994)] Under the assumptions $C 3$ and $C 4$, for every node $s$, there exists $\beta_{s} \in\left[0,1\left[\right.\right.$ such that for each agent $i$, for all $x^{i} \in F$, $x^{i} \chi_{S \backslash S+(s)}+1_{s}+\beta_{s} x^{i} \chi_{S+(s)} \succ^{i} x^{i}$.

As to the financial structure of the economy, the existence of an asset with positive returns is a classical assumption in an infinite horizon setting; it implies that the economy is "financially" connected enough. The second part of the assumption $F$ emphasizes that there is no restriction on short-selling.

\subsection{Equilibrium with debt constraints}

At every $s$, the consumers face a commodity price $p(s) \in \mathbb{R}^{L}$ (in view of the above assumptions, we can assume that $\left.p(s) \in \mathbb{R}_{+}^{L}\right)$ and an asset price $q(s) \in \mathbb{R}^{J_{0}}$. Their financial constraint at $s$ is determined by the value at $p(s)$ of their initial endowment and if $s \neq 0$ by the return paid by their preceding portfolio. Let $z_{j}^{i}(s)$ denote the number of units of the $j^{\text {th }}$ security purchased by $i$ at $s$ (if $z_{j}^{i}(s)>0$ ) or sold (if $z_{j}^{i}(s)<0$ ) and $z^{i}(s)=\left(z_{j}^{i}(s)\right)_{j \in J_{s}}$. At node $s,\left(x^{i}(s), z^{i}(s)\right)$ is the $i^{\text {th }}$ agent's consumption and portfolio vector at node $s$ and $x^{i}=\left(x^{i}(s)\right)_{s \in S}, z^{i}=\left(z^{i}(s)\right)_{s \in S}$ are the consumption and portfolio plans of $i$.

If an asset price process $q=(q(s))_{s \in S}$ is to be embedded in an equilibrium price process $(p, q)=(p(s), q(s))_{s \in S}$, it must preclude arbitrage. For every node $s$, let us 
denote by $\Phi_{s}$ the linear application $\mathbb{R}^{J_{*}} \rightarrow \mathbb{R}^{b(s)+1}$ defined by $\Phi_{s}(z(s))=\left(\begin{array}{c}-q(s) \\ R_{s}\end{array}\right) z(s)$. The asset price $q(s)$ is said arbitrage-free if there does not exist $z(s) \in \mathbb{R}^{J_{s}}$ such that $\Phi_{s}(z(s))>0$. The asset price process $q$ is said arbitrage-free if for every $s, q(s)$ is arbitrage free. The no-arbitrage condition has several consequences. The following result should be kept in mind; its easy proof will be given in the appendix.

Lemma 2. If $q$ is arbitrage-free, for every node $s$, for any vector $c_{\min } \in \mathbb{R}^{b(s)+1}$, the set $\Gamma=\left\{\varphi \in \mathbb{R}^{b(s)+1} \mid \varphi \in \operatorname{Im} \Phi_{s}\right.$ and $\left.\varphi \geq c_{\min }\right\}$ is compact.

However in an infinite horizon, taking a market system of commodity and asset price processes $(p, q)$ as given, we cannot define the budget set of agent $i$ as the set $B^{i}(p, q)=$ $\left\{\begin{array}{ll}\left(x^{i}, z^{i}\right) \in X^{i} \times \prod_{s \in S} \mathbb{R}^{J_{\bullet}} & \begin{array}{l}p(0) \cdot x^{i}(0)+q(0) \cdot z^{i}(0) \leq p(0) \cdot \omega^{i}(0) ; \\ p(s) \cdot x^{i}(s)+q(s) \cdot z^{i}(s) \leq p(s) \cdot \omega^{i}(s)+r(s) \cdot z^{i}\left(s^{-}\right) \forall s \neq 0 .\end{array}\end{array}\right\}$

As in the example given by Hernandez and Santos (1991), even if $q$ is arbitrage-free, the existence of Ponzi schemes precludes the existence of any optimal point in this kind of set. So the literature has developed several additional constraints in order to prevent the consumers from rolling over their debts indefinitely.

One of them is the notion of debt constraint introduced by Levine and Zame (1992) that limits in each node the amount of debts held by the agents at the beginning of the period.

Formally, a system of debt constraints for the agent $i$ is a function $\left.\left.D^{i}: S \rightarrow\right]-\infty, 0\right]$. The portfolio $z^{i}(s) \in \mathbb{R}^{J}$ satisfies the debt constraint $D^{i}$ at $\sigma \in s^{+}$, if $r(\sigma) \cdot z^{i}(s) \geq D^{i}(\sigma)$. If we assume for convenience that $z^{i}\left(0^{-}\right) \stackrel{\text { def }}{=} 0$, the debt constraint in node 0 will always be satisfied and the budget set $B^{i}\left(p, q, D^{i}\right)$ is now defined as follows :

$$
\left\{\begin{array}{ll}
\left(x^{i}, z^{i}\right) \in X^{i} \times \prod_{s \in S} \mathbb{R}^{J_{\bullet}} & \begin{array}{ll}
p(s) \cdot x^{i}(s)+q(s) \cdot z^{i}(s) \leq p(s) \cdot \omega^{i}(s)+r(s) \cdot z^{i}\left(s^{-}\right) & \forall s, \\
r(s) \cdot z^{i}\left(s^{-}\right) \geq D^{i}(s) & \forall s .
\end{array}
\end{array}\right\}
$$

From this definition, it is evident that $-D^{i}(s)$ denotes the maximum amount of debt that $i$ is allowed to hold at each $s$.

Equivalently, it is possible to define as Santos and Woodford (1993), for each agent $i$, a system of borrowing limits $\left.\left.M^{i}: S \rightarrow\right]-\infty, 0\right]$. The portfolio $z^{i}(s) \in \mathbb{R}^{J_{0}}$ satisfies the borrowing constraint $M^{i}$ at $s$ if $q(s) \cdot z^{i}(s) \geq M^{i}(s)$ and the budget set $B^{i}\left(p, q, M^{i}\right)$ is 
defined by :

$$
\left\{\begin{array}{l|l}
\left(x^{i}, z^{i}\right) \in X^{i} \times \prod_{s \in S} \mathbb{R}^{J_{\bullet}} & \begin{array}{ll}
p(s) \cdot x^{i}(s)+q(s) \cdot z^{i}(s) \leq p(s) \cdot \omega^{i}(s)+r(s) \cdot z^{i}\left(s^{-}\right) & \forall s, \\
q(s) \cdot z^{i}(s) \geq M^{i}(s) & \forall s .
\end{array}
\end{array}\right\}
$$

If we associate with a system of borrowing limits $M^{i}$ the system of debt constraints : $D^{i}(s)=M^{i}(s)-p(s) \cdot \omega^{i}(s)$, then $B^{i}\left(p, q, M^{i}\right) \subset B^{i}\left(p, q, D^{i}\right)$, which means that the borrowing limits are tighter than the associate debt constraints. The previous inclusion becomes an equality when the associate debt constraints satisfy looseness, one of the two properties we now define.

Indeed, as Levine and Zame (1992), we will focus our attention on debt constraints that satisfy two conditions. The first one is a consistency condition : if the debt today is bearable, there exists a portfolio plan $z^{i}(s)$ that leads to a bearable debt in each state of tomorrow. At this stage, we have to notice that this notion depends on the commodity and asset price process $(p, q)$.

Deflnition 1. The system of debt constraints $D^{i}$ is $(p, q)$-consistent at node $s$ if there exists $z^{i}(s) \in \mathbb{R}^{J}$ such that $\left\{\begin{array}{l}D^{i}(s)+p(s) \cdot \omega^{i}(s)-q(s) \cdot z^{i}(s) \geq 0, \\ \text { and for all } \sigma \in s^{+}, r(\sigma) \cdot z^{i}(s) \geq D^{i}(\sigma) .\end{array}\right.$

The system of debt constraints $D^{i}$ is said to be $(p, q)$-consistent if it is $(p, q)$-consistent at each node.

The economic interpretation of the definition is explained by the following remark.

Remark 1. For a node $s \neq 0$, an equivalent formulation of the previous definition is that for all $z^{i}\left(s^{-}\right) \in \mathbb{R}^{J_{s}-}$ such that $r(s) \cdot z^{i}\left(s^{-}\right) \geq D^{i}(s)$, there exists a plan $\left(x^{i}(s), z^{i}(s)\right) \in$ $\mathbb{R}_{+}^{L} \times \mathbb{R}^{J_{s}}$ such that $\left\{\begin{array}{l}p(s) \cdot x^{i}(s)+q(s) \cdot z^{i}(s) \leq p(s) \cdot \omega^{i}(s)+r(s) \cdot z^{i}\left(s^{-}\right) \\ \text {and for all } \sigma \in s^{+}, r(\sigma) \cdot z^{i}(s) \geq D^{i}(\sigma) .\end{array}\right.$

In other words, if the agent $i$ arrives at node $s$ with a debt $r(s) \cdot z^{i}\left(s^{-}\right)$satisfying the debt constraint in node $s$, there exists a consumption/income transfer plan at node $s$, $\left(x^{i}(s), z^{i}(s)\right)$, such that $z^{i}(s)$ finances $x^{i}(s)$ and $z^{i}(s)$ satisfies the debt constraint in every node $\sigma \in s^{+}$.

A very simple example of a $(p, q)$-consistent system of debt constraints can be found in the following proposition.

Proposition 1. Let $\left(x^{i}, z^{i}\right) \in B^{i}(p, q)$, and for every $s, D^{i}(s)=\min \left(0, r(s) \cdot z^{i}\left(s^{-}\right)\right)$. Then $D^{i}$ is a $(p, q)$-consistent system of debt constraints. 
Proof. First, since for all $s, D^{i}(s) \leq 0, D^{i}$ is a system of debt constraints. To prove consistency at node $s$, we will distinguish two cases.

- If $D^{i}(s)=0$, the portfolio $\bar{z}^{i}(s)=0_{\mathbb{R}^{J}}$ clearly satisfies

$\left\{\begin{array}{l}D^{i}(s)+p(s) \cdot \omega^{i}(s)-q(s) \cdot \bar{z}^{i}(s) \geq 0, \\ \text { and for all } \sigma \in s^{+}, r(\sigma) \cdot \bar{z}^{i}(s) \geq D^{i}(\sigma) .\end{array}\right.$

- If $D^{i}(s)<0$, it means that $D^{i}(s)=r(s) \cdot z^{i}\left(s^{-}\right)$. In this case, we define $\bar{z}^{i}(s)=z^{i}(s)$ and it follows from the spot constraint at node $s$, that

$\left\{\begin{array}{l}D^{i}(s)+p(s) \cdot \omega^{i}(s)-q(s) \cdot \bar{z}^{i}(s) \geq 0 \\ \text { and for all } \sigma \in s^{+}, r(\sigma) \cdot \bar{z}^{i}(s) \geq D^{i}(\sigma)=\min \left(0, r(\sigma) \cdot z^{i}(s)\right) .\end{array}\right.$

The second requirement is a looseness condition. If the debt today, financed by a portfolio plan $z^{i}(s)$ leads to a bearable debt in each state of tomorrow, then the debt today is bearable.

Definition 2. The system of debt constraints $D^{i}$ is $(p, q)$-loose at node $s$ if for every portfolio $z^{i}(s) \in \mathbb{R}^{J_{0}}, r(\sigma) \cdot z^{i}(s) \geq D^{i}(\sigma)$ for all $\sigma \in s^{+}$implies that $p(s) \cdot \omega^{i}(s)+D^{i}(s)-$ $q(s) \cdot z^{i}(s) \leq 0$

The system of debt constraints $D^{i}$ is $(p, q)$-loose if it is $(p, q)$-loose at every node.

In particular, if $D^{i}$ is $(p, q)$-loose at node $s$ then $D^{i}(s) \leq-p(s) \cdot \omega^{i}(s)$. The economic interpretation of Definition 2 is explained by the following remark.

Remark 2. For a node $s \neq 0$, an equivalent formulation of the previous definition is that for every plan $\left(z^{i}\left(s^{-}\right), x^{i}(s), z^{i}(s)\right) \in \mathbb{R}^{J_{0}-} \times \mathbb{R}_{+}^{L} \times \mathbb{R}^{J_{0}}$, $\left.\begin{array}{l}p(s) \cdot x^{i}(s)+q(s) \cdot z^{i}(s) \leq p(s) \cdot \omega^{i}(s)+r(s) \cdot z^{i}\left(s^{-}\right) \\ \text {and for all } \sigma \in s^{+}, r(\sigma) \cdot z^{i}(s) \geq D^{i}(\sigma) .\end{array}\right\} \Rightarrow D^{i}(s) \leq r(s) \cdot z^{i}\left(s^{-}\right)$.

In other words, if the agent $i$ arrives at node $s$, with a debt $r(s) \cdot z^{i}\left(s^{-}\right)$and uses a consumption/income transfer plan $\left(x^{i}(s), z^{i}(s)\right)$ such that $z^{i}(s)$ finances $x^{i}(s)$ and $z^{i}(s)$ satisfies the debt constraint in every node $\sigma \in s^{+}$, then the $\operatorname{debt} r(s) \cdot z^{i}\left(s^{-}\right)$satisfies the debt constraint at node $s$.

A similar requirement can be found in Santos and Woodford (1993) who define as $(p, q)$-inessential a system of borrowing limits such that the associate system of debt constraints is $(p, q)$-loose. As an analogue of Remark 2, we have : a system of borrowing limits is $(p, q)$-inessential at $s$ if and only if the borrowing constraint at $s$ is satisfied whenever the borrowing constraint and the spot budget constraint can be jointly satisfied at every 
$\sigma \in s^{+}$. Then, as an immediate consequence, any finite number of borrowing constraints may be omitted in the definition of $B^{i}\left(p, q, M^{i}\right)$.

It is worthwhile to remark that if a debt constraint is identically equal to zero, the system is consistent but in general not loose. In view of a better understanding of the properties of debt constraints, we recall that the definition of the budget set $B^{i}\left(p, q, D^{i}\right)$ involves both budget constraints and debt constraints. The following proposition will be proved in the appendix.

Proposition 2. Let $D^{i}$ be a system of debt constraints. If $q$ is arbitrage-free,

(i) There exists $\tilde{D}^{i}(p, q)$-consistent such that $B^{i}\left(p, q, \tilde{D}^{i}\right)=B^{i}\left(p, q, D^{i}\right)$ and $D^{i} \leq \tilde{D}^{i}$.

(ii) Moreover, if $D^{i}$ is $(p, q)$-loose, then there exists $\tilde{D}^{i}(p, q)$-loose and consistent such that $B^{i}\left(p, q, \tilde{D}^{i}\right)=B^{i}\left(p, q, D^{i}\right)$ and $D^{i} \leq \tilde{D}^{i}$.

The first part of this proposition was stated by Levine and Zame (1992). The interpretation is that the consistency is to be required since a $(p, q)$-consistent system of debt constraints appears to be a good representative element in the set of debt constraints that give the same budget set. Looseness (or inessentialness for borrowing limits) seems to be the most stringent requirement lightened by the next two remarks.

Remark 3. Let $M^{i}$ be a system of borrowing limits. If the system of debt constraints $D^{i}$, associated with $M^{i}$, is $(p, q)$-loose, then $B^{i}\left(p, q, D^{i}\right) \subset B^{i}\left(p, q, M^{i}\right) \subset B^{i}\left(p, q, D^{i}\right)$. If moreover $D^{i}$ is $(p, q)$-consistent, then, for every $s, M^{i}(s)=\min \left\{q(s) \cdot z^{i}(s) \mid r(\sigma) \cdot z^{i}(s) \geq\right.$ $\left.D^{i}(\sigma) \forall \sigma \in s^{+}\right\}$.

Before Remark 4, let us first introduce a definition borrowed from Levine and Zame (1992).

Definition 3. Given $p$ and $q$, the amount $d<0$ can be repaid by consumer $i$ in finite time from node $s$ if there exist $z^{i}$ and $T$ such that

$$
\left\{\begin{array}{l}
q(s) \cdot z^{i}(s) \leq p(s) \cdot \omega^{i}(s)+d \\
q(\sigma) \cdot z^{i}(\sigma) \leq p(\sigma) \cdot \omega^{i}(\sigma)+r(\sigma) \cdot z^{i}\left(\sigma^{-}\right), \forall \sigma \in S^{+}(s) \\
z^{i}(\sigma)=0 \text { if } t(\sigma) \geq t(s)+T
\end{array}\right.
$$

Let $D_{f}^{i}(s, p, q)=\inf \{d<0 \mid d$ can be repaid by consumer $i$ in finite time from node $s\}$. If for every $s, D_{f}^{i}(s, p, q)>-\infty, D_{f}^{i}(p, q)$ will be called system of finitely effective debt constraints. 
Remark 4. If the system of debt constraints $D^{i}$ is $(p, q)$-loose, then $D^{i}(s) \leq D_{f}^{i}(s, p, q)$.

Indeed, it is easily seen (by induction on $T$ ) that if the system of debt constraints $D^{i}$ is $(p, q)$-loose, then for every $s, D^{i}(s) \leq d$ whatever be $d<0$ which can be repaid in finite time from node $s$.

Moreover, if for every $s, D_{f}^{i}(s, p, q)>-\infty$, it is easily deduced from the existence of a nonrisky asset that $D_{f}^{i}(p, q)$ is $(p, q)$-loose. If $q$ is arbitrage free, it follows from Proposition 2 that $D_{f}^{i}(p, q)$ is also $(p, q)$-consistent. Hence when it is defined, the finitely effective system of debt constraints appears as the maximum of all possible systems of $(p, q)$-loose and consistent debt constraints.

Analogously, Santos and Woodford (1993) define $M_{f}^{i}(s, p, q)\left(-\pi_{\omega^{i}}(s)\right.$ in their notations) as

$M_{f}^{i}(s, p, q)=\inf \left\{\begin{array}{l|l}q(s) \cdot z^{i}(s) & \begin{array}{l}q(\sigma) \cdot z^{i}(\sigma) \leq p(\sigma) \cdot \omega^{i}(\sigma)+r(\sigma) \cdot z^{i}\left(\sigma^{-}\right) \quad \forall \sigma \in S^{+}(s), \\ z^{i}(\sigma)=0 \text { if } t(\sigma) \geq t(s)+T \text { for some } T .\end{array}\end{array}\right\}$

Obviously $D_{f}^{i}(s, p, q)=M_{f}^{i}(s, p, q)-p(s) \cdot \omega^{i}(s)$ and, in view of Remark 3 , if, for every $s$, $M_{f}^{i}(s, p, q)>-\infty, D_{f}^{i}(p, q)$ and $M_{f}^{i}(p, q)$ define the same budget set ; moreover, for every $s, M_{f}^{i}(s, p, q)=\min \left\{q(s) \cdot z^{i}(s) \mid r(\sigma) \cdot z^{i}(s) \geq D_{f}^{i}(\sigma, p, q) \forall \sigma \in s^{+}\right\}$.

Finally, assuming that no production or intertemporal storage is possible and that assets are in zero net supply, it remains to associate equilibrium definitions with the previous definitions of budget sets.

In the first one, adapted from Levine and Zame, the different systems of debt constraints are a component of the equilibrium.

Definition 4. An equilibrium with debt constraints of the economy $\mathcal{E}=\left(\left(X^{i}, \succsim^{i}, \omega^{i}\right)_{i=1}^{m}\right.$, $S, R)$ is an element $\left(\left(\bar{x}^{i}, \bar{z}^{i}, \bar{D}^{i}\right)_{i=1}^{m},(\bar{p}, \bar{q})\right)$ satisfying :

(i) For all $i,\left(\bar{x}^{i}, \bar{z}^{i}\right)$ is optimal for each agent $i$ in the budget set $B^{i}\left(\bar{p}, \bar{q}, \bar{D}^{i}\right)$;

(ii) For each $i, \bar{D}^{i}$ is a $(\bar{p}, \bar{q})$-loose and consistent system of debt constraints;

(iii) $\sum_{i=1}^{m} \bar{x}^{i} \leq \sum_{i=1}^{m} \omega^{i}$;

(iv) $\sum_{i=1}^{m} \bar{z}^{i}=0$

In the second one, adapted from Santos and Woodford who state the definition but 
do not consider the equilibrium existence problem, household specific borrowing limits are also a component of the equilibrium.

Definition 5. An equilibrium with borrowing limits of the economy $\mathcal{E}=\left(\left(X^{i}, \succsim^{i}, \omega^{i}\right)_{i=1}^{m}\right.$, $S, R)$ is an element $\left(\left(\bar{x}^{i}, \bar{z}^{i}, \bar{M}^{i}\right)_{i=1}^{m},(\bar{p}, \bar{q})\right)$ satisfying conditions (iii), (iv) of the previous definition and :

(i) For all $i,\left(\bar{x}^{i}, \bar{z}^{i}\right)$ is optimal for each agent $i$ in the budget set $B^{i}\left(\bar{p}, \bar{q}, \bar{M}^{i}\right)$.

If we add the following requirement :

(ii) For each $i, \bar{M}^{i}$ is an inessential system of borrowing limits

in view of Proposition 2 and Remark 3, the equilibrium concepts defined in 4 and 5 become equivalent.

In the third one, given the equilibrium price, the systems of debt constraints are endogenously defined.

Definition 6. A finitely effective equilibrium of the economy $\mathcal{E}=\left(\left(X^{i}, \succsim^{i}, \omega^{i}\right)_{i=1}^{m}, S, R\right)$ is an element $\left(\left(\bar{x}^{i}, \bar{z}^{i}\right)_{i=1}^{m},(\bar{p}, \bar{q})\right)$ such that $\left(\left(\bar{x}^{i}, \bar{z}^{i}, D_{f}^{i}(\bar{p}, \bar{q})\right)_{i=1}^{m},(\bar{p}, \bar{q})\right)$ is an equilibrium with debt constraints of the economy $\mathcal{E}$.

Definition 6 is borrowed from Levine and Zame. Given the previous observations and Theorem 3.3 in Hernandez and Santos (1994), it is easy to understand that it coincides with the equilibrium definition adopted by Hernandez and Santos (1994).

The two following definitions, adapted from Levine and Zame, Magill and Quinzii respectively, replace an explicit definition of the systems of debt constraints (resp. borrowing limits) at equilibrium by the overall constraint that for each consumer the real value of the debt is uniformly bounded from below. More precisely, given $p$ and $q$, let us define :

$B_{b}^{i}(p, q)=\left\{\left(x^{i}, z^{i}\right) \in B^{i}(p, q) \mid \frac{r(s) \cdot z^{i}\left(s^{-}\right)}{\|p(s)\|_{1}}\right.$ is uniformly bounded below. $\}$

$B_{\infty}^{i}(p, q)=\left\{\left(x^{i}, z^{i}\right) \in B^{i}(p, q) \mid \frac{q(s) \cdot z^{i}(s)}{\|p(s)\|_{1}}\right.$ is uniformily bounded below. $\}$

and note that $B_{\infty}^{i}(p, q) \subset B_{b}^{i}(p, q)$.

Definition 7. A bounded debt equilibrium (resp. an equilibrium with an implicit borrowing limit) of the economy $\mathcal{E}=\left(\left(X^{i}, \succsim^{i}, \omega^{i}\right)_{i=1}^{m}, S, R\right)$ is an element $\left(\left(\bar{x}^{i}, \bar{z}^{i}\right)_{i=1}^{m},(\bar{p}, \bar{q})\right)$ 
satisfying conditions (iii) and (iv) of Definition 4 and

(i) For all $i,\left(\bar{x}^{i}, \bar{z}^{i}\right)$ is optimal for agent $i$ in the budget set $B_{b}^{i}(p, q)\left(\right.$ resp. $\left.B_{\infty}^{i}(p, q)\right)$.

\subsection{Equilibrium with present value node prices}

In the definition of the individual budget sets, a second approach adapted from Hernandez and Santos (1991) and Magill and Quinzii (1994) is to add to spot budget constraints a transversality condition on the debt contracted at each period. First let us recall a classical consequence of the no-arbitrage condition. For every $s$, if $q(s)$ is arbitrage-free, there exists a vector $\lambda_{s} \in \mathbb{R}_{++}^{b(s)}, \lambda_{s}=\left(\lambda_{s}(\sigma)\right)_{\sigma \in s^{+}}$such that $q(s)=\sum_{\sigma \in s^{+}} \lambda_{s}(\sigma) r(\sigma)$. Here, we will use a more precise statement.

Lemma 3. [Schmachtenberg (1989)] and also [Hernandez and Santos (1991)]. If $q$ is a no-arbitrage asset price process for $R$, there exists $\lambda=\left(\lambda_{s}\right)_{s \in S}$ in $\mathbb{R}_{++}^{S}$, normalized by $\lambda_{0}=1$, such that for all $s$, we have $\lambda_{s} q(s)=\sum_{\sigma \in s^{+}} \lambda_{\sigma} r(\sigma)$. $\lambda$ is not necessarily unique and, in the following, $\Lambda(q)$ will denote the collection of all such $\lambda$. If $\lambda \in \Lambda(q), \lambda_{s}$ can be interpreted as the present value price of node $s$.

The definition of the individual budget sets is now based on (individualized) present value node prices. Given a market system of commodity and price processes $(p, q)$ and for each $i$, a system of present value node prices $\lambda^{i}=\left(\left(\lambda_{s}^{i}\right)\right) \in \Lambda(q)$, we define the budget set of the $i^{\text {th }}$ agent by

$$
B^{i}\left(p, q, \lambda^{i}\right)=\left\{\left(x^{i}, z^{i}\right) \in B^{i}(p, q) \text { such that } \lim _{T \rightarrow \infty} \sum_{\substack{s \in S_{T} \\ q(s) \cdot z^{i}(s) \leq 0}} \lambda_{s}^{i} q(s) \cdot z^{i}(s)=0 .\right\}
$$

Then the spot constraints at node $s$ for $s \in S^{T}$ imply that

$$
\sum_{s \in S^{T}} \lambda_{s}^{i} p(s) \cdot x^{i}(s)+\sum_{s \in S_{T}} \lambda_{s}^{i} q(s) \cdot z^{i}(s) \leq \sum_{s \in S^{T}} \lambda_{s}^{i} p(s) \cdot \omega^{i}(s) .
$$

In particular, if $\pi^{i} \stackrel{\text { def }}{=}\left(\lambda_{s}^{i} p(s)\right)_{s \in S} \in \ell_{1}(S \times L)$, with a slight abuse of notations, one has $B^{i}\left(p, q, \lambda^{i}\right) \subset\left\{x^{i} \in X^{i} \mid \pi^{i} \cdot x^{i} \leq \pi^{i} \cdot \omega^{i}\right\}$. This last set is sometimes called Arrow-Debreu budget set of agent $i$, for present value prices $\pi^{i}$.

Moreover the economic meaning of the asymptotic condition is described by the following remark. 
Remark 5. [Hernandez and Santos (1991)]. Let $\pi^{i} \stackrel{\text { def }}{=}\left(\lambda_{s}^{i} p(s)\right)_{s \in S}$. If $\pi^{i} \in \ell_{1}(S \times L)$, the asymptotic condition is equivalent to : for every $s$,

$$
\lambda_{s}^{i} q(s) \cdot z^{i}(s) \geq \sum_{\sigma \in S^{+}(s)} \lambda_{\sigma}^{i} p(\sigma) \cdot\left(x^{i}(\sigma)-\omega^{i}(\sigma)\right)=\pi^{i} \cdot\left(\left(x^{i}-\omega^{i}\right) \chi_{S^{+}(s)}\right) .
$$

In other words, if $\pi^{i} \in \ell_{1}(S \times L)$ then the definition of the budget set $B^{i}\left(p, q, \lambda^{i}\right)$ involves both spot budget constraints and an infinity of additional constraints which state that, at each node, the amount of the debt contracted by consumer $i$ should not exceed the present value of his future savings.

With such a definition of the individual budget sets, the definition of equilibrium is the following :

Definition 8. An equilibrium with present value node prices of the economy $\mathcal{E}=\left(\left(X^{i}\right.\right.$, $\left.\left.\succsim^{i}, \omega^{i}\right)_{i=1}^{m}, S, R\right)$ is an element $\left(\left(\bar{x}^{i}, \bar{z}^{i}, \bar{\lambda}^{i}\right)_{i=1}^{m},(\bar{p}, \bar{q})\right)$ satisfying :

(i) For all $i,\left(\bar{x}^{i}, \bar{z}^{i}\right)$ is optimal for each agent $i$ in the budget set $B^{i}\left(\bar{p}, \bar{q}, \bar{\lambda}^{i}\right)$;

(ii) For each $i, \bar{\lambda}^{i} \in \Lambda(q)$;

(iii) $\sum_{i=1}^{m} \bar{x}^{i} \leq \sum_{i=1}^{m} \omega^{i}$;

(iv) $\sum_{i=1}^{m} \bar{z}^{i}=0$.

In the previous definition, we do not require the present value price $\pi^{i}=\left(\bar{\lambda}_{s}^{i} \bar{p}(s)\right)_{s \in S}$ to belong to $\ell_{1}(S \times L)$ though this condition is an assumption of Remark 5. In fact, under the additionnal assumption $C 5$, the condition $\pi^{i} \in \ell_{1}(S \times L)$ is useless (cf. Remark 8 in Section 4).

Definition 8, adapted from Hernandez and Santos (1991), does not actually coincide with the definition given by Magill and Quinzii (1994). In their definition of the individual budget sets associated with a market system of commodity and asset price processes $(p, q)$, and for each $i$ a system of individualized present value node prices $\lambda^{i}$, Magill and Quinzii require from an agent to be neither lender nor borrower at infinity. Moreover in the spirit of the subgame perfection, they require this condition on every subtree of the initial eventtree. Outside of equilibrium, it makes sense to prevent every agent from being borrower at infinity but not necessarily lender at infinity. In view of this remark, we define the 
alternative budget set :

$$
B_{M Q}^{i}\left(p, q, \lambda^{i}\right)=\left\{\left(x^{i}, z^{i}\right) \in B^{i}(p, q) \mid \liminf _{T \rightarrow+\infty} \sum_{\sigma \in S_{T} \cap S(s)} \lambda_{\sigma}^{i} q(\sigma) \cdot z^{i}(\sigma) \geq 0 \quad \forall s \in S\right\} .
$$

This budget set is related to the previous one by the following remark.

Remark 6. $B^{i}\left(p, q, \lambda^{i}\right) \subset B_{M Q}^{i}\left(p, q, \lambda^{i}\right)$; moreover if $\pi^{i} \stackrel{\text { def }}{=}\left(\lambda_{s}^{i} p(s)\right)_{s \in S} \in \ell_{1}(S \times L)$, one has also $B_{M Q}^{i}\left(p, q, \lambda^{i}\right) \subset\left\{x^{i} \in X^{i} \mid \pi^{i} \cdot x^{i} \leq \pi^{i} \cdot \omega^{i}\right\}$.

This definition of the budget sets leads to the corresponding concept of equilibrium.

Definition 9. A MQ-equilibrium with present value node prices of the economy $\mathcal{E}=\left(\left(X^{i}\right.\right.$, $\left.\left.\succsim^{i}, \omega^{i}\right)_{i=1}^{m}, S, R\right)$ is an element $\left(\left(\bar{x}^{i}, \bar{z}^{i}, \bar{\lambda}^{i}\right)_{i=1}^{m},(\bar{p}, \bar{q})\right)$ satisfying :

(i) For all $i,\left(\bar{x}^{i}, \bar{z}^{i}\right)$ is optimal for each agent $i$ in the budget set $B_{M Q}^{i}\left(\bar{p}, \bar{q}, \bar{\lambda}^{i}\right)$; and conditions (ii), (iii), (iv), of Definition 8.

\section{Existence of equilibria with debt constraints}

In this section, we prove that every asset price process that precludes arbitrage can be embedded in an equilibrium with debt constraints of the economy $\mathcal{E}$. From now and in the whole paper, an arbitrage-free asset price process $q$ will be assumed to be given.

Theorem 1. Under the assumptions $C 1$ to $C_{4}$ and $F$ and if $q$ precludes arbitrage, there exists an equilibrium with debt constraints $\left(\left(\bar{x}^{i}, \bar{z}^{i}, \bar{D}^{i}\right)_{i=1}^{m}, \bar{p}, q\right)$ of the economy $\mathcal{E}=\left(\left(X^{i}\right.\right.$, $\left.\left.\succsim^{i}, \omega^{i}\right)_{i=1}^{m}, S, R\right)$ satisfying $\bar{p}(s)>0$, for all $s$. If for every $s, \bar{M}^{i}(s)=\bar{D}^{i}(s)+\bar{p}(s) \cdot \omega^{i}(s)$, $\left(\left(\bar{x}^{i}, \bar{z}^{i}, \bar{M}^{i}\right)_{i=1}^{m}, \bar{p}, q\right)$ is an equilibrium with borrowing limits of $\mathcal{E}$

Proof. First let us fix a particular $\lambda \in \Lambda(q)$. The main steps of our proof are as follows. If $T$ denotes a time horizon, the first step is to obtain a particular general equilibrium with incomplete markets of the $T$-truncated economy $\mathcal{E}(T)$ with the same characteristics as $\mathcal{E}$, in which agents are constrained to stop trading at date $T$. The second step is to construct the implicit debt constraints of this $T$-truncated economy. The third step consists in establishing uniform bounds (in $T$ ) on the parameters of equilibria. Thus, it is possible in a fourth step to take an appropriate limit of these parameters. The fifth step is to prove that the system of debt constraints obtained at the limit is loose and consistent, and the final step shows that this limit is an equilibrium for the infinite horizon economy. 
Step 1. Let us fix a time horizon $T$. If $p^{T} \in \mathbb{R}_{+}^{S^{T} \times L}$ is the commodity price process, then the budget set of agent $i$ in the truncated economy $\mathcal{E}(T)$ is classically defined by $B^{i, T}\left(p^{T}, q\right)=$

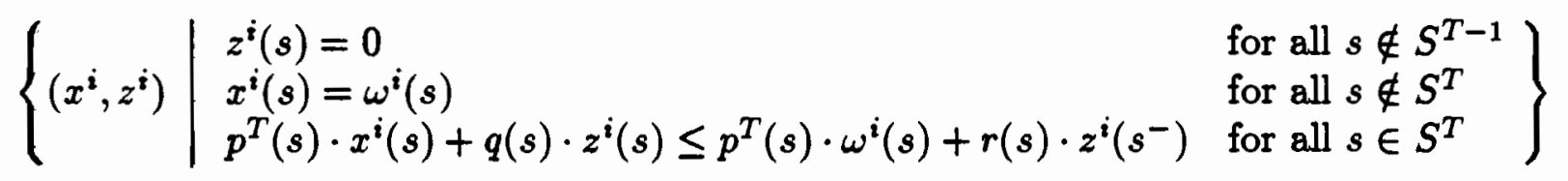

Even though the consumption-portfolio process of an agent be defined over the whole event-tree, a $T$-truncated economy is essentially a finite horizon economy with $T+1$ periods since the consumption-portfolio process of an agent is fixed after date $T$. As it is classical, we will not distinguish the finite dimensional vector $p^{T}$ and its natural embedding in the infinite dimensional space $\mathbb{R}_{+}^{S \times L}$ defined by $p^{T}(s)=0$ if $s \notin S^{T}$. The context will make it clear.

With a slight abuse of notation, we recall that

$$
B^{i, T}\left(p^{T}, q\right) \subset\left\{x^{i} \in X^{i} \mid \begin{array}{ll}
x^{i}(s)=\omega^{i}(s) & \text { if } s \notin S^{T} \\
\sum_{s \in S^{T}} \lambda_{s} p^{T}(s) \cdot\left(x^{i}(s)-\omega^{i}(s)\right) \leq 0
\end{array}\right\} .
$$

If we use the following notation $\pi^{T}=\left(\pi^{T}(s)\right)_{s \in S} \stackrel{\text { def }}{=}\left(\lambda_{s} p^{T}(s)\right)_{s \in S}$, the previous set can be equivalently rewritten as

and $B^{i, T}\left(p^{T}, q\right) \subset B^{i, T}\left(\pi^{T}\right)$

$$
B^{i, T}\left(\pi^{T}\right) \stackrel{\text { def }}{=}\left\{\begin{array}{l|l}
x^{i} \in X^{i} & \begin{array}{l}
x^{i}(s)=\omega^{i}(s) \text { if } s \notin S^{T} \\
\pi^{T} \cdot\left(x^{i}-\omega^{i}\right) \leq 0
\end{array}
\end{array}\right\}
$$

According to Florenzano and Gourdel (1994), the finite-horizon economy $\mathcal{E}(T)$ has an equilibrium $\left(\left(\bar{x}^{i, T}, \bar{z}^{i, T}\right)_{i=1}^{m}, \bar{p}^{T}, q\right)$ satisfying :

(i) $\bar{x}^{1, T}$ is optimal in the budget set $B^{1, T}\left(\bar{\pi}^{T}\right)$ where $\bar{\pi}^{T}(s)=\lambda_{s} \bar{p}^{T}(s)$.

(ii) For all $i=1, \ldots, m,\left(\bar{x}^{i, T}, \bar{z}^{i, T}\right)$ is optimal for agent $i$ in the budget set $B^{i, T}\left(\bar{p}^{T}, q\right)$.

(iii) $\sum_{i=1}^{m} \bar{x}^{i, T}=\sum_{i=1}^{m} \omega^{i}$

(iv) $\sum_{i=1}^{m} \bar{z}^{i, T}=0$.

(v) $\bar{p}^{T} \in \mathbb{R}_{+}^{S^{T} \times L}$ and for all node $s \in S^{T}, \bar{p}^{T}(s) \neq 0$.

With no loss of generality, we can assume that the price $\bar{p}^{T}$ is normalized by $\sum_{s \in S^{T}} \lambda_{s}\left\|\bar{p}^{T}(s)\right\|_{1}=1$. Note that this normalization requires an adaptation of the portfolios that with an abuse of notation, we will still denote by $\bar{z}^{i, T}$. So, the claims (i) to (v) are still verified for this normalized equilibrium and we can add the additional one : 
(vi) $\left\|\bar{\pi}^{T}\right\|_{1}=1$ or equivalently $\bar{\pi}^{T} \cdot 1=1$ (with $1 \in \mathbb{R}^{S \times L}$ defined by $1(s, \ell)=1$ ).

Step 2. As Levine and Zame (1992) pointed out, in the finite horizon model, it is possible to associate with the above equilibrium an implicit system of debt constraints. It can be defined by

$$
\bar{D}^{i, T}(s)=\inf \left\{\bar{p}^{T}(s) \cdot\left(x^{i}(s)-\omega^{i}(s)\right)+q(s) \cdot z^{i}(s)\right\}
$$

where the infimum is taken among all $\left(x^{i}, z^{i}\right)$ belonging to $B^{i, T}\left(\bar{p}^{T}, q\right)$. The notion of implicit debt constraint means that

$$
\begin{aligned}
& \left\{\begin{array}{l|l}
\left(x^{i}, z^{i}\right) & \begin{array}{ll}
z^{i}(s)=0 & \text { for all } s \notin S^{T-1} \\
x^{i}(s)=\omega^{i}(s) & \text { for all } s \notin S^{T} \\
\bar{p}^{T}(s) \cdot x^{i}(s)+q(s) \cdot z^{i}(s) \leq \bar{p}^{T}(s) \cdot \omega^{i}(s)+r(s) \cdot z^{i}\left(s^{-}\right) & \text {for all } s \in S^{T}
\end{array}
\end{array}\right\} \\
& =\left\{\begin{array}{ll}
\left(x^{i}, z^{i}\right) & \begin{array}{ll}
r(s) \cdot z^{i}\left(s^{-}\right) \geq \bar{D}^{i, T}(s) & \text { for all } s \in S^{T} \\
z^{i}(s)=0 & \text { for all } s \notin S^{T-1} \\
x^{i}(s)=\omega^{i}(s) & \text { for all } s \notin S^{T} \\
\bar{p}^{T}(s) \cdot x^{i}(s)+q(s) \cdot z^{i}(s) \leq \bar{p}^{T}(s) \cdot \omega^{i}(s)+r(s) \cdot z^{i}\left(s^{-}\right) & \text {for all } s \in S^{T}
\end{array}
\end{array}\right\}
\end{aligned}
$$

We emphasize that this construction could have been done for any price $p^{T}$, not only for the equilibrium price. As a consequence of the no-arbitrage condition on $q$, the main result at this step is the following proposition to be proved in the appendix :

Proposition 3. $\bar{D}^{i, T}$ is $\left(\bar{p}^{T}, q\right)$-loose and consistent. Moreover $\bar{D}^{i, T}(s)=D_{f}^{i}\left(s, \bar{p}^{T}, q\right)$ $\forall s \in S^{T-1}$.

Step 3. In this step, we will prove that the parameters $\left(\left(\bar{x}^{i, T}(s), \Phi_{s}\left(\bar{z}^{i, T}(s)\right)\right.\right.$, $\left.\left.\bar{D}^{i, T}(s)\right)_{i=1}^{m}, \bar{p}^{T}(s)\right)_{s \in S}$ of the $T$-horizon equilibrium are bounded in $T$.

- Bounds on $\bar{x}^{i, T}(s, \ell)$. Since $\bar{x}^{i, T}(s, \ell) \geq 0$ and $\sum_{i=1}^{m} \bar{x}^{i, T}(s, \ell)=\sum_{i=1}^{m} \omega^{i}(s, \ell) \leq M$, where $M=\left\|\sum_{i=1}^{m} \omega^{i}\right\|_{\infty}$. It follows that $0 \leq \bar{x}^{i, T}(s, \ell) \leq M$, for all $(s, \ell) \in S \times L$ and for each agent $i$.

- Bounds on $\bar{p}^{T}(s, \ell)$. First we recall that $\bar{p}^{T}(s, \ell) \geq 0$, hence our normalization $1=\sum_{(s, \ell) \in S^{T} \times L} \lambda_{s} \bar{p}^{T}(s, \ell)$ leads to $0 \leq \bar{p}^{T}(s, \ell) \leq 1 / \lambda_{s}$, for all $(s, \ell) \in S \times L$.

- Bounds on $\Phi_{s}\left(\bar{z}^{i, T}(s)\right)$. We first prove by contraposition that the first component of $\Phi_{s}\left(\bar{z}^{i, T}(s)\right)$, which is equal to $q(s) \cdot \bar{z}^{i, T}(s)$, is bounded. If it is not true, there exists an agent $i$, a node $s$ and a subsequence denoted by $T_{n}$ such that $\left|q(s) \cdot \bar{z}^{i, T_{n}}(s)\right| \rightarrow+\infty$ when $T_{n} \rightarrow+\infty$. From condition (iv) in the definition of equilibrium, we can deduce the existence 
of an agent $j$ such that $q(s) \cdot \bar{z}^{j, T_{n}}(s) \rightarrow+\infty$ when $T_{n} \rightarrow+\infty$. The agent $j$ is lender at node $s$. But for $T_{n}$ large enough, one has $\bar{p}^{T_{n}}(s) \cdot 1_{s} \leq\left(1-\beta_{s}\right) q(s) \cdot \bar{z}^{j, T_{n}}(s)$ since $\beta_{s}<1$. This implies that the consumption process $\left(\bar{x}^{j, T_{n}} \chi_{S \backslash S+(s)}+1_{s}+\beta_{s} \bar{x}^{j, T_{n}} \chi_{S+(s)}\right)$ sustained by the portfolio process $\left(\bar{z}^{j, T_{n}} \chi_{S \backslash S(s)}+\beta_{s} \bar{z}^{j, T_{n}} \chi_{S(s)}\right)$ is in the budget set $B^{j, T_{n}}\left(\bar{p}^{T_{n}}, q\right)$. Since by Lemma 1 , it is preferred by agent $j$ to the consumption $\bar{x}^{j, T_{n}}$, this contradicts the definition of equilibrium.

By local non-satiation, one has for each agent $i$, for all nodes $s$, for all $\sigma \in s^{+}$, $r(\sigma) \cdot \bar{z}^{i, T}(s)=q(\sigma) \cdot \bar{z}^{i, T}(\sigma)+\bar{p}^{T}(\sigma) \cdot\left(\bar{x}^{i, T}(\sigma)-\omega^{i}(\sigma)\right)$. Hence, from the bounds established previously on $\bar{x}^{i, T}(\sigma), \bar{p}^{T}(\sigma)$ and $q(\sigma) \cdot \bar{z}^{i, T}(\sigma)$, one deduces that $r(\sigma) \cdot \bar{z}^{i, T}(s)$ is also bounded. This proves that $\Phi_{s}\left(\bar{z}^{i, T}(s)\right)$ is bounded.

- Bounds on $\bar{D}^{i, T}(s)$. First we recall that $\bar{D}^{i, T}(s) \leq 0$. Let us define on $S$ the system of debt constraint $D^{i, T}$ by :

$$
D^{i, T}(s)=\frac{-\bar{\pi}^{T} \cdot\left(\omega^{i} \chi_{S(s)}\right)}{\lambda_{s}}=-\sum_{\sigma \in S(s)}\left(\frac{\lambda_{\sigma}}{\lambda_{s}}\right) \bar{p}^{T}(\sigma) \cdot \omega^{i}(\sigma)
$$

Clearly, $D^{i, T}(s)=-\bar{p}^{T}(s) \cdot \omega^{i}(s)$ if $s \in S_{T}$ and $D^{i, T}(s)=0$ if $s \notin S^{T}$. We first prove that $D^{i, T}$ is $\left(\bar{p}^{T}, q\right)$-loose. Consider a node $s \in S$. If $s \notin S^{T-1}$, looseness of $D^{i, T}$ at node $s$ follows from the no-arbitrage condition. If $s \in S^{T-1}$, let us consider a portfolio $z^{i}(s) \in \mathbb{R}^{J_{s}}$ such that for all node $\sigma \in s^{+}, r(\sigma) \cdot z^{i}(s) \geq D^{i, T}(\sigma)$. By definition of $D^{i, T}$, one has for all node $\sigma \in s^{+}, \lambda_{\sigma} r(\sigma) \cdot z^{i}(s) \geq-\bar{\pi}^{T} \cdot\left(\omega^{i} \chi_{S(\sigma)}\right)$. This implies that $\sum_{\sigma \in s^{+}} \lambda_{\sigma} r(\sigma) \cdot z^{i}(s) \geq$ $-\bar{\pi}^{T} \cdot\left(\sum_{\sigma \in s^{+}} \omega^{i} \chi_{S(\sigma)}\right)=-\bar{\pi}^{T} \cdot\left(\omega^{i} \chi_{S+(s)}\right)$. Since $\lambda$ satisfies conditions of Lemma 3 , one thus gets $\lambda_{s} q(s) \cdot z^{i}(s) \geq-\bar{\pi}^{T} \cdot\left(\omega^{i} \chi_{S+(s)}\right)$. We recall that $S(s)=S^{+}(s) \cup\{s\}$ and consequently $\lambda_{s} q(s) \cdot z^{i}(s) \geq-\pi^{T} \cdot\left(\omega^{i} \chi s(s)\right)+\lambda_{s} \bar{p}^{T}(s) \cdot \omega^{i}(s)$, i.e., $D^{i, T}(s)+\bar{p}^{T}(s) \cdot \omega^{i}(s)-q(s) \cdot z^{i}(s) \leq 0$. Hence $D^{i, T}$ is $\left(\bar{p}^{T}, q\right)$-loose at node $s$.

Since $D^{i, T}$ is $\left(\bar{p}^{T}, q\right)$-loose, we can apply Propositions 2, 3 and Remark 4 to get the inequality $\bar{D}^{i, T} \geq D^{i, T}$. Moreover, it follows from the normalization of $\bar{\pi}^{T}$ that $D^{i, T}(s) \geq$ $-M / \lambda_{s}$. Hence, we deduce that for all $i$, all nodes $s, 0 \geq \bar{D}^{i, T}(s) \geq-M / \lambda_{s}$.

Step 4. Let $b a(S \times L)=\left(\ell_{\infty}(S \times L)\right)^{*}$ denote the norm dual of $\ell_{\infty}(S \times L)$ consisting of bounded finitely additive set functions on $S \times L$ and let $\|\cdot\|_{b a}$ denotes the norm of $b a(S \times L)$. For any $T$, the price $\bar{\pi}^{T}$ can be viewed as an element of $(b a(S \times L))_{+}$such that $\bar{\pi}^{T} \cdot 1=\left\|\bar{\pi}^{T}\right\|_{b a}=1$. Let $\sigma\left(b a, \ell_{\infty}\right)$ denote the weak-star topology. We recall that according to Alaoglu's theorem, the unit ball of $b a$ is $\sigma\left(b a, \ell_{\infty}\right)$-compact.

In view of the bounds established previously, we can apply Tychonov's Theorem to get the existence of a directed set $(\Theta, \geq)$ such that the subnet $\left(\left(\bar{x}^{i, T^{\theta}},\left(\Phi_{s}\left(\bar{z}^{i, T^{\theta}}(s)\right)\right)_{s \in S}\right.\right.$, 
$\left.\left.\bar{D}^{i, T^{\theta}}\right)_{i=1}^{m}, \bar{p}^{T^{\theta}}\right)_{\theta \in \Theta}$ converges for the product topology and $\left(\bar{\pi}^{T^{\theta}}\right)_{\theta \in \Theta}$ converges for the $\sigma\left(b a, \ell_{\infty}\right)$-topology. Hence, we get the existence of an element $\left(\left(\bar{x}^{i},\left(\bar{\varphi}^{i}(s)\right)_{s \in S}, \bar{D}^{i}\right)_{i=1}^{m}, \bar{p}\right)$ in the set $\left(\mathbb{R}_{+}^{S \times L} \times \prod_{s \in S} \mathbb{R}^{b(s)+1} \times \mathbb{R}_{-}^{S}\right)^{m} \times \mathbb{R}_{+}^{S \times L}$ and an element $\bar{\pi}$ in $(b a(S \times L))_{+}$such that

$$
\left(\left(\bar{x}^{i, T^{\theta}},\left(\Phi_{s}\left(\bar{z}^{i, T^{\theta}}(s)\right)\right)_{s \in S}, \bar{D}^{i, T^{\theta}}\right)_{i=1}^{m}, \bar{p}^{T^{\theta}}\right) \rightarrow\left(\left(\bar{x}^{i},\left(\bar{\varphi}^{i}(s)\right)_{s \in S}, \bar{D}^{i}\right)_{i=1}^{m}, \bar{p}\right)
$$

for the product topology and

$$
\bar{\pi}^{T^{\theta}} \rightarrow \bar{\pi} \text { for the } \sigma\left(b a, \ell_{\infty}\right) \text { topology with } \bar{\pi} \cdot 1=1 .
$$

Let us remark that it follows from the bounds established in Step 3 that $\bar{x}^{i} \in X^{i}$. Indeed, one has for all $T$ and each $i, 0 \leq \bar{x}^{i, T}(s, l) \leq M$. Moreover, it follows from the same inequality that one can assume that $\bar{x}^{i, T^{\theta}} \rightarrow \bar{x}^{i}$ in the weak topology $\sigma\left(\ell_{\infty}(S \times L), \ell_{1}(S \times\right.$ L)).

Since for all node $s, \operatorname{Im} \Phi_{s}$ is a closed subset of $\mathbb{R}^{b(s)+1}$ and since $\Phi_{s}\left(\bar{z}^{i, T^{\theta}}(s)\right) \rightarrow \bar{\varphi}^{i}(s)$, one deduces that $\bar{\varphi}^{i}(s) \in \operatorname{Im} \Phi_{s}$. I.e., for all $i=2, \ldots, m$, there exists some $\bar{z}^{i}(s) \epsilon$ $\mathbb{R}^{J_{s}}$ such that $\Phi_{s}\left(\bar{z}^{i, T^{\theta}}(s)\right) \rightarrow \Phi_{s}\left(\bar{z}^{i}(s)\right)$. Using the Cass' trick, if we let $\bar{z}^{1}=\sum_{i=2}^{m} \bar{z}^{i}$, one deduces from the property (iv) of the finite horizon equilibrium that $\Phi_{s}\left(\bar{z}^{1, T^{\theta}}(s)\right) \rightarrow$ $\Phi_{s}\left(\bar{z}^{1}(s)\right)$. Note that $\bar{z}^{i, T^{\theta}}(s)$ does not necessarily converge to $\bar{z}^{i}(s)$ since redundant assets may exist, in particular the subnet $\left(\bar{z}^{i, T^{\theta}}(s)\right)_{\theta \in \Theta}$ may be unbounded.

Step 5. We want to prove that for each agent $i$, the system of debt constraint $\bar{D}^{i}$ is $:(\bar{p}, q)$-loose and consistent.

- $\bar{D}^{i}$ is $(\bar{p}, q)$-loose. Let us consider an agent $i$, a node $s$ and a portfolio $z^{i}(s) \in \mathbb{R}^{J_{\bullet}}$ such that $r(\sigma) \cdot z^{i}(s) \geq \bar{D}^{i}(\sigma)$ for all $\sigma \in s^{+}$.

We want to prove that $\bar{D}^{i}(s)+\bar{p}(s) \cdot \omega^{i}(s)-q(s) \cdot z^{i}(s) \leq 0$. To this end, we will use the existence of a non risky asset at node $s$, denoted by $\xi(s)$. Hence for all $\varepsilon>0$, one has $r(\sigma) \cdot\left(z^{i}(s)+\varepsilon \xi(s)\right)>\bar{D}^{i}(\sigma)$ for all $\sigma \in s^{+}$. Since there is a finite number of strict inequalities, there exists $\bar{\theta}$ such that for all $\theta \geq \bar{\theta}$, one has $r(\sigma) \cdot\left(z^{i}(s)+\varepsilon \xi(s)\right)>\bar{D}^{i, T^{\theta}}(\sigma)$ for all $\sigma \in s^{+}$. Consequently, by $\left(\bar{p}^{T^{\theta}}, q\right)$-looseness of the implicit debt constraint $\bar{D}^{i, T^{\theta}}$, one has $\bar{D}^{i, T^{\theta}}(s)+\bar{p}^{T^{\theta}}(s) \cdot \omega^{i}(s)-q(s) \cdot\left(z^{i}(s)+\varepsilon \xi(s)\right) \leq 0$. At the limit when $\theta$ goes to infinity, one gets $\bar{D}^{i}(s)+\bar{p}(s) \cdot \omega^{i}(s)-q(s) \cdot\left(z^{i}(s)+\varepsilon \xi(s)\right) \leq 0$. It suffices to take the limit when $\varepsilon$ tends to 0 of the previous inequality to end the proof.

- $\bar{D}^{i}$ is $(\bar{p}, q)$-consistent. Let us consider an agent $i$ and a node $s$. We first recall that for all $\theta$, the implicit debt constraint $\bar{D}^{i, T^{\theta}}$ is $\left(\bar{p}^{T^{\theta}}, q\right)$-consistent, hence there exists a 
portfolio $z^{i, T^{\theta}}(s)$ such that

$$
\left\{\begin{array}{l}
\bar{D}^{i, T^{\theta}}(s)+\bar{p}^{T^{\theta}}(s) \cdot \omega^{i}(s)-q(s) \cdot z^{i, T^{\theta}}(s) \geq 0 \\
\text { for all } \sigma \in s^{+}, r(\sigma) \cdot z^{i, T^{\theta}}(s) \geq \bar{D}^{i, T^{\theta}}(\sigma)
\end{array}\right.
$$

Note that the portfolio $z^{i, T^{\theta}}(s)$ is defined without any reference to the portfolio $\bar{z}^{i, T^{\theta}}(s)$. The system can be equivalently rewritten as

$$
\Phi_{s}\left(z^{i, T^{\theta}}(s)\right) \geq\left(\begin{array}{c}
-\bar{D}^{i, T^{\theta}}(s)-\bar{p}^{T^{\theta}}(s) \cdot \omega^{i}(s) \\
\left(\bar{D}^{i, T^{\theta}}(\sigma)\right)_{\sigma \in s^{+}}
\end{array}\right)
$$

Hence the net $\left(\Phi_{s}\left(z^{i, T^{\theta}}(s)\right)\right)_{\theta \in \Theta}$ is bounded below. It follows from Lemma 2 that with no loss of generality, one can assume that $\Phi_{s}\left(z^{i, T^{\theta}}(s)\right)$ tends to $\Phi_{s}\left(z^{i}(s)\right)$ for some portfolio $z^{i}(s) \in \mathbb{R}^{J_{\bullet}}$. By passing to limit in the previous inequalities, we get

$$
\left\{\begin{array}{l}
\bar{D}^{i}(s)+\bar{p}(s) \cdot \omega^{i}(s)-q(s) \cdot z^{i}(s) \geq 0 \\
\text { for all } \sigma \in s^{+}, r(\sigma) \cdot z^{i}(s) \geq \bar{D}^{i}(\sigma)
\end{array}\right.
$$

This completes the proof.

Step 6. It remains to prove that $\left(\left(\bar{x}^{i}, \bar{z}^{i}, \bar{D}^{i}\right)_{i=1}^{m}, \bar{p}\right)$ is an equilibrium with debt constraints of the economy $\mathcal{E}$.

It is trivial to verify that individual consumption and income transfer plans belong to the individual budget set at each node $s$, and that consumption plans and income transfer plans are socially feasible.

- For each $i, \bar{x}^{i} \in B^{i}\left(\bar{p}, q, \bar{D}^{i}\right)$. Indeed, by passing to limit in the spot constraints, one has for each node $s$,

$$
\begin{aligned}
& \left\{\begin{array}{l}
\bar{p}(s) \cdot \bar{x}^{i}(s)+q(s) \cdot \bar{z}^{i}(s)=r(s) \cdot \bar{z}^{i}\left(s^{-}\right)+\bar{p}(s) \cdot \omega^{i}(s), \\
r(s) \cdot \bar{z}^{i}\left(s^{-}\right) \geq \bar{D}^{i}(s) .
\end{array}\right. \\
& \text { - } \sum_{i=1}^{m} \bar{x}^{i}=\sum_{i=1}^{m} \omega^{i} \\
& \text { - } \sum_{i=1}^{m} \bar{z}^{i}=0
\end{aligned}
$$

- We want to prove that consumption $\bar{x}^{i}$ is optimal in $B^{i}\left(\bar{p}, q, \bar{D}^{i}\right)$. To this end, the following claims will first prove that for all node $s, \bar{p}(s)>0$. 
Claim 1. $a^{1} \succ^{1} \bar{x}^{1} \Rightarrow \bar{\pi} \cdot a^{1} \geq \bar{\pi} \cdot \omega^{1}$.

We recall that $\bar{x}^{1, T^{9}} \rightarrow \bar{x}^{1}$ for the weak topology, and $a^{1} \chi_{S^{N}} \rightarrow a^{1}$ for the Mackey topology. Since the relation $\hbar^{1}$ is Mackey continuous, there exists some $N$ such that $a^{1} \chi_{S^{N}} \succ^{1} \bar{x}^{1}$. By the same argument, there exists some $\bar{\theta} \in \Theta$ such that for all $\theta \geq \bar{\theta}$, one has $a^{1} \chi_{S^{N}} \succ^{1} \bar{x}^{1, T^{\theta}}$ and we can suppose that $T^{\bar{\theta}} \geq N$. Hence by monotonicity, one obtains for all $\theta \geq \bar{\theta}, a^{1} \chi_{S^{T^{\theta}}} \succ^{1} \bar{x}^{1, T^{\theta}}$. Since $a^{1} \chi_{S^{T^{\theta}}}+\omega^{1} \chi_{S \backslash S^{T^{\theta}}}$ is in the finite horizon consumption set and preferred by agent 1 to the equilibrium consumption $\bar{x}^{1, T^{\theta}}$, it follows from the property (i) of the finite horizon equilibrium that $\bar{\pi}^{T^{\theta}} \cdot\left(a^{1} \chi_{S^{r^{\theta}}}+\omega^{1} \chi_{S \backslash S^{T^{\theta}}}\right)>$ $\bar{\pi}^{T^{\theta}} \cdot \bar{x}^{1, T^{\theta}}$. But $\bar{\pi}^{T^{\theta}} \cdot\left(a^{1} \chi_{S^{T^{\theta}}}+\omega^{1} \chi_{S \backslash S^{T^{\theta}}}\right)=\bar{\pi}^{T^{\theta}} \cdot a^{1} \chi_{S^{T^{\theta}}}$ and by local non-satiation, one obtains $\bar{\pi}^{T^{\theta}} \cdot \bar{x}^{1, T^{\theta}}=\bar{\pi}^{T^{\theta}} \cdot \omega^{1}$. Hence, there exists $\bar{\theta}$ such that for all $\theta \geq \bar{\theta}, \bar{\pi}^{T^{\theta}} \cdot a^{1} \geq$ $\bar{\pi}^{T^{\theta}} \cdot a^{1} \chi_{S^{T^{\theta}}}>\bar{\pi}^{T^{\theta}} \cdot \omega^{1}$. At the limit when $\theta$ goes to infinity, one gets $\bar{\pi} \cdot a^{1} \geq \bar{\pi} \cdot \omega^{1}$.

Since $\bar{\pi} \in b a(S \times L)$ and $\bar{\pi} \geq 0$, we can apply the Yosida-Hewitt theorem to get the existence of some $\bar{\pi}_{c} \in \ell_{1}^{+}(S \times L)$ such that $\bar{\pi}$ and $\bar{\pi}_{c}$ coincide on "finite" elements of $\ell_{\infty}(S \times L)$. For any $y \in \ell_{\infty}(S \times L)$ which has only a finite number of non-zero components, one has $\bar{\pi}_{c} \cdot y=\bar{\pi} \cdot y$.

Claim 2. $a^{1} \succ^{1} \bar{x}^{1} \Rightarrow \bar{\pi}_{c} \cdot a^{1} \geq \bar{\pi} \cdot \omega^{1}$.

The proof is similar to the one of Claim 1. Since $a^{1} \succ^{1} \bar{x}^{1}$, there exists some integer $N$, such that $a^{1} \chi_{S^{N}} \succ^{1} \bar{x}^{1}$. Hence, we can apply the first claim to get the following inequality : $\bar{\pi} \cdot a^{1} \chi_{S^{N}} \geq \bar{\pi} \cdot \omega^{1}$. By definition of $\bar{\pi}_{c}$, one deduces $\bar{\pi}_{c} \cdot a^{1} \chi_{S^{N}} \geq \bar{\pi} \cdot \omega^{1}$. This assertion together with the positivity of $\bar{\pi}_{c}$ gives us $\bar{\pi}_{c} \cdot a^{1} \geq \bar{\pi} \cdot \omega^{1}$.

Claim 3. $a^{1} \succ^{1} \bar{x}^{1} \Rightarrow \bar{\pi}_{c} \cdot a^{1}>\bar{\pi} \cdot \omega^{1}$.

First, we recall that $\omega^{1} \in \operatorname{int}\left(\ell_{\infty}^{+}(S \times L)\right)$, since $\bar{\pi} \cdot 1=1$, one deduces that $\bar{\pi} \cdot \omega^{1}>0$. Since $a^{1} \succ^{1} \bar{x}^{1}$, by continuity of $\succsim^{1}$, there exists some real number $\alpha<1$ such that $\alpha a^{1} \succ^{1} \bar{x}^{1}$. It follows from Claim 2 that $\bar{\pi}_{c} \cdot\left(\alpha a^{1}\right)=\alpha \bar{\pi}_{c} \cdot a^{1} \geq \bar{\pi} \cdot \omega^{1}$. Hence, one obtains $\bar{\pi}_{c} \cdot a^{1} \geq \frac{1}{\alpha} \bar{\pi} \cdot \omega^{1}>\bar{\pi} \cdot \omega^{1}$.

Claim 4. $\bar{\pi}_{c} \cdot \bar{x}^{1} \leq \bar{\pi} \cdot \omega^{1}$.

Let us fix an integer $N$, one has $\bar{\pi}^{T^{\theta}} \cdot\left(\bar{x}^{1, T^{\theta}} \chi_{S^{N}}\right) \leq \bar{\pi}^{T^{\theta}} \cdot \bar{x}^{1, T^{\theta}}=\bar{\pi}^{T^{\theta}} \cdot \omega^{1}$ for all $\theta$. This can be equivalently rewritten as $\sum_{s \in S^{N}} \lambda_{s} \bar{p}^{T^{\theta}}(s) \cdot \bar{x}^{1, T^{\theta}}(s) \leq \bar{\pi}^{T^{\theta}} \cdot \omega^{1}$. Since there is only a finite number of terms on the left side, one gets at the limit in $\theta, \sum_{s \in S^{N}} \lambda_{s} \bar{p}(s) \cdot \bar{x}^{1}(s) \leq$ $\bar{\pi} \cdot \omega^{1}$. We have proved that for all $N, \bar{\pi}_{c} \cdot\left(\bar{x}^{1} \chi_{S^{N}}\right) \leq \bar{\pi} \cdot \omega^{1}$. At the limit in $N$, one gets 
$\bar{\pi}_{c} \cdot \bar{x}^{1} \leq \bar{\pi} \cdot \omega^{1}$

Claim 5. $\bar{p}(s)>0$ for each node $s$.

Finally, it follows from Assumption $C 3$, that $\bar{x}^{1}+1_{s} \succ^{1} \bar{x}^{1}$. One can apply Claim 3 to get the following inequality : $\bar{\pi}_{c} \cdot\left(\bar{x}^{1}+1_{s}\right)>\bar{\pi} \cdot \omega^{1}$. This assertion, together with Claim 4 gives us $\bar{\pi}_{c} \cdot \bar{x}^{1}+\bar{\pi}_{c} \cdot 1_{s}>\bar{\pi} \cdot \omega^{1} \geq \bar{\pi}_{c} \cdot \bar{x}^{1}$. Consequently, one obtains : $\bar{\pi}_{c} \cdot 1_{s}=\lambda_{s}\|\bar{p}\|_{1}>0$ which establishes that $\bar{p}(s)>0$.

For each $i$, we will now prove by contraposition that $\bar{x}^{i}$ is optimal in $B^{i}\left(\bar{p}, q, \bar{D}^{i}\right)$.

Let us suppose that $a^{i} \succ^{i} \bar{x}^{i}$ and $\left(a^{i}, z^{i}\right) \in B^{i}\left(\bar{p}, q, \bar{D}^{i}\right)$ for some portfolio plan $z^{i}$. By Mackey continuity of the preferences, there exists some real number $\alpha<1$ such that $\alpha a^{i} \succ^{i} \bar{x}^{i}$. We can use the same argument as in Claim 1 of Step 6 to get the existence of some integer $N$ and some $\bar{\theta} \in \Theta$ such that for all $\theta \geq \bar{\theta}$, one has $\left(\alpha a^{i} \chi_{S^{N}}\right) \succ^{i} \bar{x}^{i, T^{\theta}}$. Since $\left(a^{i}, z^{i}\right) \in B^{i}\left(\bar{p}, q, \bar{D}^{i}\right)$, we have in particular the following inequalities :

$$
\begin{cases}\bar{p}(s) \cdot a^{i}(s)+q(s) \cdot z^{i}(s) \leq \bar{p}(s) \cdot \omega^{i}(s)+r(s) \cdot z^{i}\left(s^{-}\right) & \text {for all } s \in S^{N} \\ r(s) \cdot z^{i}\left(s^{-}\right) \geq \bar{D}^{i}(s) & \text { for all } s \in S^{N+1} .\end{cases}
$$

We recall that a consequence of the $(\bar{p}, q)$-looseness of $\bar{D}^{i}$ is that $\bar{D}^{i}(s) \leq-\bar{p}(s) \cdot \omega^{i}(s)$ for all nodes $s$. This assertion together with $\bar{p}(s)>0$ and $\omega^{i}(s) \gg 0$ implies that $\bar{p}(s)$. $\omega^{i}(s)>0$ and $\bar{D}^{i}(s)<0$. Since $\alpha<1$, the last remark together with the previous system of inequalities implies that

$$
\begin{cases}\bar{p}(s) \cdot\left(\alpha a^{i}(s)\right)+q(s) \cdot\left(\alpha z^{i}(s)\right)<\bar{p}(s) \cdot \omega^{i}(s)+r(s) \cdot\left(\alpha z^{i}\left(s^{-}\right)\right) & \text {for all } s \in S^{N} \\ r(s) \cdot\left(\alpha z^{i}\left(s^{-}\right)\right)>\bar{D}^{i}(s) & \text { for all } s \in S^{N+1} .\end{cases}
$$

Since there is only a finite number of strict inequalities, one deduces the existence of an integer $\bar{T}>N$ such that for all $T \geq \bar{T}$, one has

$$
\begin{cases}\bar{p}^{T}(s) \cdot\left(\alpha a^{i}(s)\right)+q(s) \cdot\left(\alpha z^{i}(s)\right)<\bar{p}^{T}(s) \cdot \omega^{i}(s)+r(s) \cdot\left(\alpha z^{i}\left(s^{-}\right)\right) & \text {for all } s \in S^{N} \\ r(s) \cdot\left(\alpha z^{i}\left(s^{-}\right)\right)>\bar{D}^{i, T}(s) & \text { for all } s \in S^{N+1}\end{cases}
$$

With no loss of generality, we may suppose that $\bar{T}>T^{\bar{\theta}}$. But it follows from the consistency of the implicit debt constraint $\bar{D}^{i, T}$ that there exists some portfolio plan $\tilde{z}^{i, T}$ such that $\tilde{z}^{i, T}$ is equal to zero from date $T$ and $\tilde{z}^{i, T}$ and $\alpha z^{i}$ coincide on $S^{N}$. By construction, one has $\left(\alpha a^{i} \chi_{S^{N}}, \tilde{z}^{i, T}\right) \in B^{i, T}\left(\bar{p}^{T}, q\right)$. Since for some $T$ large enough, one also has $\alpha a^{i} \chi_{S^{N}} \succ^{i} \bar{x}^{i, T}$, this contradicts the definition of the equilibrium consumption $\bar{x}^{i, T}$. 
The last assertion in Theorem 1 is a consequence of Remark 3.

At this stage, it may be asked what are the properties of the previous equilibrium under the additional Assumption $C 5$, formulated by Magill and Quinzii and used in their existence result of an equilibrium with node prices. An answer is given in the following result.

Theorem 2. Let $\left(\left(\bar{x}^{i}, \bar{z}^{i}, \bar{D}^{i}\right)_{i=1}^{m}, \bar{p}, q\right)$ be an equilibrium with debt constraints of the economy $\mathcal{E}$. Under Assumption $C 5,\left(\left(\bar{x}^{i}, \bar{z}^{i}\right)_{i=1}^{m}, \bar{p}, q\right)$ is a finitely effective equilibrium, a bounded debt equilibrium and an equilibrium with an implicit borrowing limit.

The proof is based on the following result to be proved in the appendix.

Proposition 4. Given $(p, q)$, let $D^{i}$ be a consistent and uniformely bounded below (in real value) system of debt constraints. Then every amount of debt $d, 0>d>D^{i}\left(s_{0}\right)$ can be repaid by $i$ in finite time from node $s_{0}$. Consequently, one has $D^{i}\left(s_{0}\right) \geq D_{f}^{i}\left(s_{0}, p, q\right)$.

Proof of Theorem 2. Let $\left(\left(\bar{x}^{i}, \bar{z}^{i}, \bar{D}^{i}\right)_{i=1}^{m}, \bar{p}, q\right)$ be an equilibrium with debt constraints of the economy $\mathcal{E}$. We first prove that for each $i$ :

$$
B_{\infty}^{i}(\bar{p}, q) \subset B_{b}^{i}(\bar{p}, q) \subset B^{i}\left(\bar{p}, q, D_{f}^{i}(\bar{p}, q)\right) \subset B^{i}\left(\bar{p}, q, \bar{D}^{i}\right)
$$

In effect, let $\left(x^{i}, z^{i}\right) \in B_{b}^{i}(\bar{p}, q)$ and for every $s, D^{i}(s)=\min \left(0, r(s) \cdot \bar{z}^{i}\left(s^{-}\right)\right)$. The system $D^{i}$ is $(\bar{p}, q$ )-consistent (cf. Proposition 1) and uniformly bounded below. It follows from Proposition 4 that $\left.D_{f}^{i}(s, \bar{p}, q) \leq D^{i}(s) \leq r(s) \cdot \bar{z}^{i}\left(s^{-}\right)\right)$, which proves the second inclusion. The looseness of $\bar{D}^{i}$ together with Remark 4 lead to $\bar{D}^{i}(s) \leq D_{f}^{i}(s, \bar{p}, q)$ which implies the last inclusion. The first inclusion was already observed.

Under Assumption $C 5$, let us now consider a node $s$ and an agent $i$. Let $\left(\tilde{x}_{s}^{i}, \tilde{z}_{s}^{i}\right)$ be the consumption/portfolio plan defined by $\left(\bar{x}^{i} \chi_{S \backslash S^{+}(s)}+1_{s}+\beta \bar{x}^{i} \chi_{S^{+}(s)}, \bar{z}^{i} \chi_{S \backslash S(s)}+\beta \bar{z}^{i} \chi_{S(s)}\right)$. Since by assumption $C 5, \tilde{x}_{s}^{i} \succ^{i} \bar{x}^{i}$, one deduces that $\left(\tilde{x}_{s}^{i}, \tilde{z}_{s}^{i}\right) \notin B^{i}\left(\bar{p}, q, \bar{D}^{i}\right)$. Since $\left(\tilde{x}_{s}^{i}, \tilde{z}_{s}^{i}\right)$ obviously satisfies the debt constraint in every node and the spot constraint in every node $s^{\prime} \neq s$, it follows that the spot constraint in node $s$ is not satisfied. This implies that $\bar{p}(s) \cdot\left(x^{i}(s)+1_{s}\right)+q(s) \cdot \beta \bar{z}^{i}(s)>\bar{p}(s) \cdot \omega^{i}(s)+r(s) \cdot \bar{z}^{i}\left(s^{-}\right)$. This inequality together with the spot constraint satisfied by $\left(\bar{x}^{i}, \bar{z}^{i}\right)$ at node $s$ leads to $\bar{p}(s) \cdot 1_{s}+q(s) \cdot\left(\beta \bar{z}^{i}(s)\right)>q(s) \cdot \bar{z}^{i}(s)$. One thus gets $q(s) \cdot \bar{z}^{i}(s)<\|\bar{p}(s)\|_{1} /(1-\beta)$. Since this inequality holds for all $i$, and recalling that $\sum_{i=1}^{m} \bar{z}^{i}(s)=0$, it follows that $q(s) \cdot \bar{z}^{i}(s)>-(m-1)\|\bar{p}(s)\|_{1} /(1-\beta)$ for all nodes $s$, and all agents $i$. This proves that $\left(\bar{x}^{i}, \bar{z}^{i}\right) \in B_{\infty}^{i}(\bar{p}, q)$ and ends the proof. 


\section{Debt constraints versus node prices}

Let us there emphasize that, as proved in Section 3, the existence of an equilibrium with debt constraints, compatible with the given arbitrage-free asset price process $q$, does not require Assumption C5. Under Assumption $C 5$, such an equilibrium is a finitely effective and a bounded debt equilibrium. We will prove in this section that with the same additional assumption, the same given arbitrage-free asset price process can be embedded in an equilibrium with node prices. According to the role played by Assumption $C 5$ in the existence of an equilibrium with node prices (Assumption $C 5$ was formulated by Magill and Quinzii and is used in their existence result), the link between both approaches cannot be reduced to a simple equivalence.

Actually from (individualized) node prices, it is easy to build (individualized) debt constraints; a more precise statement will be given in Proposition 5. Under the additional assumption $C 5$, the converse construction is only shown to be possible at an equilibrium point. Theorem 4 and 5 will make it clear. Finally, our main result, Theorem 6 , is to prove that six apparently quite distinct approaches in the definition of equilibrium give in fact under the assumptions $C 1-C 5$ and $F$ the same equilibrium allocations and prices.

Proposition 5. Assume that $\lambda^{i}$ is a system of present value node prices, satisfying the conditions of Lemma 9 , such that $\pi^{i} \stackrel{\text { def }}{=}\left(\lambda_{s}^{i} p(s)\right)_{s \in S} \in \ell_{1}(S \times L)$. Then there exists a $(p, q)$ loose and consistent system of debt constraints $\tilde{D}^{i}$ such that $B^{i}\left(p, q, \lambda^{i}\right)=B^{i}\left(p, q, \tilde{D}^{i}\right)$.

Proof. Let us define the system of debt constraints $D^{i}$ by : for each node $s$,

$$
D^{i}(s)=\frac{-\pi^{i} \cdot\left(\omega^{i} \chi_{S(s)}\right)}{\lambda_{s}^{i}}=-\sum_{\sigma \in S(s)}\left(\frac{\lambda_{\sigma}^{i}}{\lambda_{s}^{i}}\right) p(\sigma) \cdot \omega^{i}(\sigma)
$$

Since $\pi^{i} \in \ell_{1}(S \times L)$, and since for every node $s, \lambda_{s}^{i}>0, D^{i}$ is well defined. We first prove that $D^{i}$ is $(p, q)$-loose. Indeed let us consider a node $s$ and a portfolio $z^{i}(s)$ such that for every node $\sigma \in s^{+}, r(\sigma) \cdot z^{i}(s) \geq D^{i}(\sigma)$. By definition of $D^{i}$, one has for every node $\sigma \in s^{+}, \lambda_{\sigma}^{i} r(\sigma) \cdot z^{i}(s) \geq-\pi^{i} \cdot\left(\omega^{i} \chi_{S(\sigma)}\right)$. This implies that $\sum_{\sigma \in s^{+}} \lambda_{\sigma}^{i} r(\sigma) \cdot z^{i}(s) \geq-\pi^{i}$. $\left(\sum_{\sigma \in s^{+}} \omega^{i} \chi_{S(\sigma)}\right)=-\pi^{i} \cdot\left(\omega^{i} \chi_{S^{+}(s)}\right)$. Since $\lambda^{i}$ satisfies, the conditions of Lemma 3 , one thus gets $\lambda_{s}^{i} q(s) \cdot z^{i}(s) \geq-\pi^{i} \cdot\left(\omega^{i} \chi_{S+(s)}\right)$. We recall that $S(s)=S^{+}(s) \cup\{s\}$ and consequently $\lambda_{s}^{i} q(s) \cdot z^{i}(s) \geq-\pi^{i} \cdot\left(\omega^{i} \chi_{S(s)}\right)+\lambda_{s}^{i} p(s) \cdot \omega^{i}(s)$, i.e., $D^{i}(s)+p(s) \cdot \omega^{i}(s)-q(s) \cdot z^{i}(s) \leq 0$. Hence $D^{i}$ is $(p, q)$-loose at each node $s$.

Let us prove that $B^{i}\left(p, q, \lambda^{i}\right) \subset B^{i}\left(p, q, D^{i}\right)$. Indeed, let us consider $\left(x^{i}, z^{i}\right)$ in $B^{i}\left(p, q, \lambda^{i}\right)$. It follows from the budget constraint in node $s$ that $\lambda_{s}^{i} r(s) \cdot z^{i}\left(s^{-}\right) \geq \lambda_{s}^{i} p(s) \cdot$ 
$\left(x^{i}(s)-\omega^{i}(s)\right)+\lambda_{s}^{i} q(s) \cdot z^{i}(s)$. This inequality together with the one of Remark 4 leads to $\lambda_{s}^{i} r(s) \cdot z^{i}\left(s^{-}\right) \geq \sum_{\sigma \in S(s)} \lambda_{\sigma}^{i} p(\sigma) \cdot\left(x^{i}(\sigma)-\omega^{i}(\sigma)\right)$. Since $x^{i} \geq 0$, one obtains $\lambda_{s}^{i} r(s) \cdot z^{i}\left(s^{-}\right) \geq-\pi^{i} \cdot\left(\omega^{i} \chi_{S(s)}\right)$. By definition of $D^{i}$, the debt constraint in node $s$ is satisfied and $\left(x^{i}, z^{i}\right) \in B^{i}\left(p, q, D^{i}\right)$.

Let us prove that conversely $B^{i}\left(p, q, D^{i}\right) \subset B^{i}\left(p, q, \lambda^{i}\right)$. Indeed, let us consider $\left(x^{i}, z^{i}\right)$ in $B^{i}\left(p, q, D^{i}\right)$. Since $D^{i}$ is $(p, q)$-loose, one has for all node $s, D^{i}(s)+p(s) \cdot \omega^{i}(s)-$ $q(s) \cdot z^{i}(s) \leq 0$. This implies that $\lambda_{s}^{i} q(s) \cdot z^{i}(s) \geq-\pi^{i} \cdot\left(\omega^{i} \chi_{S+(s)}\right)$ and

$$
0 \geq \sum_{\substack{s \in S_{T} \\ q(s) \cdot z^{i}(s) \leq 0}} \lambda_{s}^{i} q(s) \cdot z^{i}(s) \geq-\pi^{i} \cdot\left(\omega^{i} \chi_{S \backslash S^{T}}\right) .
$$

Since the term on the right side of the previous inequality tends to zero in the limit in $T$, one obtains $\left(x^{i}, z^{i}\right)$ in $B^{i}\left(p, q, \lambda^{i}\right)$.

Finally, we can apply Proposition 2 to get the existence of a $(p, q)$-loose and consistent system of debt constraint $\tilde{D}^{i}$ such that $B^{i}\left(p, q, D^{i}\right)=B^{i}\left(p, q, \tilde{D}^{i}\right)$.

The previous construction can be achieved for any price system $(p, q)$. If we apply Proposition 5 to the equilibrium prices, we obtain the following result.

Theorem 3. Let $\left(\left(\bar{x}^{i}, \bar{z}^{i}, \bar{\lambda}^{i}\right)_{i=1}^{m}, \bar{p}, q\right)$ be an equilibrium with present value node prices of the economy $\mathcal{E}=\left(\left(X^{i}, \succsim^{i}, \omega^{i}\right)_{i=1}^{m}, S, R\right)$. If for each $i, \bar{\pi}^{i} \stackrel{\text { def }}{=}\left(\bar{\lambda}_{s}^{i} \bar{p}(s)\right)_{s \in S} \in \ell_{1}(S \times L)$, then there exists some debt constraints $\bar{D}^{i}$ such that $\left(\left(\bar{x}^{i}, \bar{z}^{i}, \bar{D}^{i}\right)_{i=1}^{m}, \bar{p}, q\right)$ is an equilibrium with debt constraints of the economy $\mathcal{E}$.

Under the additional Assumption C5, conversely one also has :

Theorem 4. Let $\left(\left(\bar{x}^{i}, \bar{z}^{i}, \bar{D}^{i}\right)_{i=1}^{m}, \bar{p}, q\right)$ be an equilibrium with debt constraints of the economy $\mathcal{E}=\left(\left(X^{i}, \succsim^{i}, \omega^{i}\right)_{i=1}^{m}, S, R\right)$. Under the assumptions $C 1-C 5$ and $F$, there exists some present value node prices $\left(\bar{\lambda}^{i}\right)_{i=1}^{m}$ such that $\left(\left(\bar{x}^{i}, \bar{z}^{i}, \bar{\lambda}^{i}\right)_{i=1}^{m}, \bar{p}, q\right)$ is an equilibrium with present value node prices of the economy $\mathcal{E}$. Moreover $\left(\left(\bar{x}^{i}, \bar{z}^{i}, \bar{\lambda}^{i}\right)_{i=1}^{m}, \bar{p}, q\right)$ is also a $M Q$-equilibrium with present value node prices of the economy $\mathcal{E}$.

Proof. Let us consider for any agent $i$, and for any time horizon $T$, the set $B_{T}^{i}(\bar{p}, q)$ defined by

$$
\left\{\begin{array}{l|l}
x^{i} \in \mathbb{R}^{S^{T} \times L}\left|\exists z^{i} \in \prod_{s \in S^{T}} \mathbb{R}^{J_{*}}\right| \begin{array}{l}
\bar{p}(s) \cdot x^{i}(s)+q(s) \cdot z^{i}(s) \leq \bar{p}(s) \cdot \omega^{i}(s)+r(s) \cdot z^{i}\left(s^{-}\right) \\
\forall s \in S^{T} \\
-r(s) \cdot z^{i}\left(s^{-}\right) \leq 0 \quad \forall s \in S_{T+1}
\end{array}
\end{array}\right\} .
$$


As it is classical, we will not distinguish the finite dimensional set $B_{T}^{i}(\bar{p}, q)$ and its natural embedding in the infinite dimensional space $\ell_{\infty}(S \times L) \times \prod_{s \in S} \mathbb{R}^{J_{\bullet}}$ defined by $(x(s), z(s))=$ $\left(\omega^{i}(s), 0\right)$ if $s \notin S^{T}$. The context will make it clear. Note that the sets $B_{T}^{i}(\bar{p}, q)$ are nonempty and convex and that $B_{T}^{i}(\bar{p}, q) \subset B_{T+1}^{i}(\bar{p}, q)$, for all $T \geq 1$. We can now define the two convex subsets of $\ell_{\infty}(S \times L)$ defined by

$$
\begin{aligned}
& U^{i}=\left\{x^{i} \in \ell_{\infty}^{+}(S \times L) \mid x^{i} \succ \bar{x}^{i}\right\} \\
& A^{i}=\operatorname{co}\left\{\left\{\bar{x}^{i}\right\} \cup \bigcup_{T \geq 1} B_{T}^{i}(\bar{p}, q) .\right\}
\end{aligned}
$$

This set can be equivalently rewritten as

$$
A^{i}=\left\{x^{i} \in \ell_{\infty}(S \times L) \mid \exists\left(\mu, \tilde{x}^{i}\right) \in[0,1] \times \bigcup_{T \geq 1} B_{T}^{i}(\bar{p}, q) \text { such that } x^{i}=\mu \bar{x}^{i}+(1-\mu) \tilde{x}^{i}\right\} .
$$

Claim 1. $U^{i} \cap A^{i}=\emptyset$.

Let us consider a point $x^{i}$ in the intersection. First, we remark that it follows from the definition of $U^{i}$ that $x^{i}$ is non-negative. Since $x^{i} \in A^{i}$, there exists some integer $T$, a point $\left(\tilde{x}^{i}, \tilde{z}^{i}\right) \in B_{T}^{i}(\bar{p}, q)$ and a real number of $[0,1]$ such that $x^{i}=\mu \bar{x}^{i}+(1-$ $\mu) \tilde{x}^{i}$. We can associate the portfolio plan $z^{i}=\mu \bar{z}^{i}+(1-\mu) \tilde{z}^{i}$. We want to prove that $\left(x^{i}, z^{i}\right) \in B^{i}\left(\bar{p}, q, \bar{D}^{i}\right)$. Clearly, the spot constraints are satisfied in every node. Moreover $\left(x^{i}, z^{i}\right)$ satisfies the debt constraints $\bar{D}^{i}$ in all nodes $s \notin S^{T}$. Since $x^{i}$ is non-negative, it follows from the $(\bar{p}, q)$-looseness of the debt constraint $\bar{D}^{i}$ that $\left(x^{i}, z^{i}\right)$ satisfies the debt constraints in all nodes. Hence $\left(x^{i}, z^{i}\right) \in B^{i}\left(\bar{p}, q, \bar{D}^{i}\right) \cap U^{i}$, which contradicts the definition of equilibrium with debt constraints.

The assumption $C 4$ implies that $\bar{x}^{i}+1 \in \operatorname{int}\left(U^{i}\right)$. Hence, we can apply the HahnBanach theorem to get the existence of some non-zero element $P^{i}$ in $\left(\ell_{\infty}(S \times L)\right)_{+}^{\prime}=$ $(b a(S \times L))_{+}$such that for all $\left(x^{i}, y^{i}\right) \in A^{i} \times U^{i}$, one has $P^{i} \cdot x^{i} \leq P^{i} \cdot y^{i}$. Since $\bar{x}^{i} \in A^{i}$, and since by local non-satiation $\bar{x}^{i} \in \operatorname{cl}\left(U^{i}\right)$, one deduces that for all $\left(x^{i}, y^{i}\right) \in A^{i} \times U^{i}$, one has $P^{i} \cdot x^{i} \leq P^{i} \cdot \bar{x}^{i} \leq P^{i} \cdot y^{i}$. Since $P^{i}$ is in $b a(S \times L)_{+}$, we can apply the Yosida-Hewitt theorem to get the existence of some $\bar{\pi}^{i} \in \ell_{1}^{+}(S \times L)$ such that $P^{i}$ and $\pi^{i}$ coincide on "finite" elements of $\ell_{\infty}(S \times L)$.

We first claim that $a^{i} \succ \bar{x}^{i} \Rightarrow \pi^{i} \cdot a^{i} \geq P^{i} \cdot \bar{x}^{i}$. Indeed, we recall that $a^{i} \chi_{S^{N}} \rightarrow a^{i}$ for the Mackey topology and consequently, there exists some integer $N$ such that $a^{i} \chi_{S^{N}} \succ^{i} \bar{x}^{i}$. 
It follows that $P^{i} \cdot a^{i} \chi_{S^{N}} \geq P^{i} \cdot \bar{x}^{i}$. By definition of $\pi^{i}$, one deduces that $\pi^{i} \cdot a^{i} \chi_{S^{N}} \geq P^{i} \cdot \bar{x}^{i}$. This assertion together with the positivity of $\pi^{i}$ leads to $\pi^{i} \cdot a^{i} \geq P^{i} \cdot \bar{x}^{i}$.

By local non satiation of the preferences, we deduce from the last claim that $\pi^{i} \cdot \bar{x}^{i} \geq$ $P^{i} \cdot \bar{x}^{i}$. This implies that $\pi^{i} \cdot \bar{x}^{i}=P^{i} \cdot \bar{x}^{i}$ by definition of $\pi^{i}$.

Claim 2. $\bar{x}^{i}$ is optimal for agent $i$ in the set $\left\{x^{i} \in X^{i} \mid \pi^{i} \cdot x^{i} \leq \pi^{i} \cdot \bar{x}^{i}\right\}$.

We first prove that $\pi^{i} \cdot \bar{x}^{i}>0$. Since $\left(\omega^{i}, 0\right) \in A^{i}$, one deduces that $P^{i} \cdot \omega^{i} \leq \pi^{i} \cdot \bar{x}^{i}$. Recalling that $P^{i}$ is a non-zero element of $(b a(S \times L))_{+}$and $\omega^{i} \in \operatorname{int}\left(\ell_{\infty}^{+}(S \times L)\right)$, it follows that $P^{i} \cdot \omega^{i}>0$ and consequently $\pi^{i} \cdot \bar{x}^{i}>0$. Let us now consider $x^{i} \in X^{i}$ such that $x^{i} \succ^{i} \bar{x}^{i}$. It follows from the continuity of the preferences that there exists some real number $\alpha<1$ such that $\alpha x^{i} \succ^{i} \bar{x}^{i}$. Hence we deduce from the previous results that $\pi^{i} \cdot\left(\alpha x^{i}\right) \geq \pi^{i} \cdot \bar{x}^{i}$. One thus gets $\pi^{i} \cdot x^{i} \geq \frac{1}{\alpha} \pi^{i} \cdot \bar{x}^{i}>\pi^{i} \cdot \bar{x}^{i}$.

In particular, we deduce from Assumption $C 4$ and Claim 2 that $\pi^{i}(s)>0$, for every node $s$.

Let us now construct the node prices $\bar{\lambda}^{i}$ corresponding to the present value prices $\pi^{i}$. For any time horizon $T$, for all $x^{i} \in B_{T}^{i}(\bar{p}, q)$ one has $P^{i} \cdot x^{i} \leq \pi^{i} \cdot \bar{x}^{i}$. This implies that $\pi^{i} \cdot x^{i} \chi_{S^{T}}+P^{i} \cdot \omega^{i} \chi_{S \backslash S^{T}} \leq \pi^{i} \cdot \bar{x}^{i}$, and consequently $\pi^{i} \cdot x^{i} \chi_{S^{T}} \leq \pi^{i} \cdot \bar{x}^{i}$. We have proved that the obviously consistent system of inequalities defining $B_{T}^{i}(\bar{p}, q)$ implies that $\sum_{s \in S^{T}} \pi^{i}(s) \cdot x^{i}(s) \leq \pi^{i} \cdot \bar{x}^{i}$. Hence we can apply the non-homogeneous Farkas' lemma (cf. Rockafellar (1970), Theorem 22.3) to get the existence of some $\left(\left(\lambda_{s}^{i, T}\right)_{s \in S^{T}},\left(\mu_{s}^{i, T}\right)_{s \in S_{T+1}}\right) \in$ $\mathbb{R}_{+}^{S^{T}} \times \mathbb{R}_{+}^{S_{T+1}}$ such that :

$$
\begin{aligned}
& \pi^{i}(s)=\lambda_{s}^{i, T} \bar{p}(s) \text { for all } s \text { in } S^{T} \\
& \lambda_{s}^{i, T} q(s)=\sum_{\sigma \in s^{+}} \lambda_{\sigma}^{i, T} r(\sigma) \text { for all } s \text { in } S^{T-1} \\
& \lambda_{s}^{i, T} q(s)=\sum_{\sigma \in s^{+}} \mu_{\sigma}^{i, T} r(\sigma) \text { for all } s \text { in } S_{T} \\
& \text { and } \pi^{i} \cdot \bar{x}^{i} \geq \sum_{s \in S^{T}} \lambda_{s}^{i, T} \bar{p}(s) \cdot \omega^{i}(s) .
\end{aligned}
$$

Recalling that $\pi^{i}(s)>0$ for all $s \in S$, we deduce from (1) that the real number $\lambda_{s}^{i, T}$ is positive and independent of $T$. This allow the construction of some $\bar{\lambda}^{i}$ in $\mathbb{R}_{++}^{S}$ satisfying $\pi^{i}(s)=\bar{\lambda}_{s}^{i} \bar{p}(s)$ for all $s$ in $S$; moreover using (2), one also has $\bar{\lambda}_{s}^{i} q(s)=\sum_{\sigma \in \theta^{+}} \bar{\lambda}_{\sigma}^{i} r(\sigma)$ for all $s$ in $S$. Since $\pi^{i} \cdot \omega^{i} \leq \pi^{i} \cdot \bar{x}^{i}$, one deduces that $B^{i}\left(\bar{p}, q, \bar{\lambda}^{i}\right) \subset\left\{x^{i} \in X^{i} \mid \pi^{i} \cdot x^{i} \leq \pi^{i} \cdot \omega^{i}\right\} \subset$ $\left\{x^{i} \in X^{i} \mid \pi^{i} \cdot x^{i} \leq \pi^{i} \cdot \bar{x}^{i}\right\}$. Recalling that $\bar{x}^{i}$ is optimal in the last set, it suffices to prove that $\left(\bar{x}^{i}, \bar{z}^{i}\right) \in B^{i}\left(\bar{p}, q, \bar{\lambda}^{i}\right)$ to get the optimality of $\left(\bar{x}^{i}, \bar{z}^{i}\right)$ in $B^{i}\left(\bar{p}, q, \bar{\lambda}^{i}\right)$. 
Claim 3. $\left(\bar{x}^{i}, \bar{z}^{i}\right) \in B^{i}\left(\bar{p}, q, \bar{\lambda}^{i}\right)$.

In the proof of Theorem 2, we have established that if $\left(\bar{x}^{i}, \bar{z}^{i}\right)$ is optimal in $B^{i}\left(\bar{p}, q, \bar{D}^{i}\right)$ then, for all nodes $s$ and all agents $i$ one has $q(s) \cdot \bar{z}^{i}(s)>-(m-1)\|\bar{p}(s)\|_{1} /(1-\beta)$ for all nodes $s$. These inequalities lead to

$$
\sum_{\substack{s \in S_{T} \\ q(s) \cdot \bar{z}^{i}(s) \leq 0}} \bar{\lambda}_{s}^{i} q(s) \cdot \bar{z}^{i}(s)>-\frac{m-1}{1-\beta} \sum_{\substack{s \in S_{T} \\ q(s) \cdot \bar{z}^{i}(s) \leq 0}} \bar{\lambda}_{s}^{i}\|\bar{p}(s)\|_{1} .
$$

Hence we deduce that

$$
0 \geq \sum_{\substack{s \in S_{T} \\ q(s) \cdot \bar{z}^{i}(s) \leq 0}} \bar{\lambda}_{s}^{i} q(s) \cdot \bar{z}^{i}(s)>-\frac{m-1}{1-\beta} \sum_{s \in S_{T}} \bar{\lambda}_{s}^{i}\|\bar{p}(s)\|_{1}=-\frac{m-1}{1-\beta} \pi^{i} \cdot 1 \chi_{S_{T}} .
$$

Since $\pi^{i}$ is in $\ell_{1}(S \times L)$, the right term tends to zero, when $T$ goes to infinity. Hence one has $\left(\bar{x}^{i}, \bar{z}^{i}\right) \in B^{i}\left(\bar{p}, q, \bar{\lambda}^{i}\right)$.

Moreover $\left(\bar{x}^{i}, \bar{z}^{i}\right)$ is optimal in $B_{M Q}^{i}\left(\bar{p}, q, \bar{\lambda}^{i}\right)$. Indeed, it follows from Remark 5 that $B^{i}\left(\bar{p}, q, \bar{\lambda}^{i}\right) \subset B_{M Q}^{i}\left(\bar{p}, q, \bar{\lambda}^{i}\right) \subset\left\{x^{i} \in X^{i} \mid \pi^{i} \cdot x^{i} \leq \pi^{i} \cdot \omega^{i}\right\} \subset\left\{x^{i} \in X^{i} \mid \pi^{i} \cdot x^{i} \leq \pi^{i} \cdot \bar{x}^{i}\right\}$. Recalling that $\bar{x}^{i}$ is optimal in the largest set and that $\left(\bar{x}^{i}, \bar{z}^{i}\right) \in B^{i}\left(\bar{p}, q, \bar{\lambda}^{i}\right)$, one gets the conclusion of the last assertion of Theorem 4.

Remark 7. It is worthwhile to note that the previous proof could be applied to the particular equilibrium with debt constraints constructed in the proof of Theorem 1. This shows that, given any $\lambda \in \Lambda(q)$, there exists an equilibrium with node prices (and also a $M-Q$ equilibrium) in which the first consumer uses precisely $\lambda$.

Theorem 5. Let $\left(\left(\bar{x}^{i}, \bar{z}^{i}, \bar{\lambda}^{i}\right)_{i=1}^{m}, \bar{p}, q\right)$ be a MQ-equilibrium with present value node prices of the economy $\mathcal{E}=\left(\left(X^{i}, \succsim^{i}, \omega^{i}\right)_{i=1}^{m}, S, R\right)$. Under the assumptions $C 1-C 5$ and $F$, there exists some present value node prices $\left(\tilde{\lambda}^{i}\right)_{i=1}^{m}$ such that $\left(\left(\bar{x}^{i}, \bar{z}^{i}, \tilde{\lambda}^{i}\right)_{i=1}^{m}, \bar{p}, q\right)$ is an equilibrium with present value node prices of the economy $\mathcal{E}$, satisfying for each $i, \tilde{\pi}^{i} \stackrel{\text { def }}{=}\left(\tilde{\lambda}_{s}^{i} \bar{p}(s)\right)_{s \in S} \in$ $\ell_{1}(S \times L)$.

Proof. The proof of Theorem 5 is similar to the one of Theorem 4. Let us consider for any agent $i$, the two convex subsets of $\ell_{\infty}(S \times L)$ defined by

$$
A^{i}=\left\{x^{i} \in \ell_{\infty}(S \times L)\left|\exists z^{i} \in \prod_{s \in S} \mathbb{R}^{J_{s}}\right| \begin{array}{ll}
\mid \bar{p}(s) \cdot\left(x^{i}(s)-\omega^{i}(s)\right)+q(s) \cdot z^{i}(s) \leq r(s) \cdot z^{i}\left(s^{-}\right) \\
\forall s \in S ; \\
\liminf _{T \rightarrow+\infty} \sum_{\sigma \in S_{T} \cap S(s)} \bar{\lambda}_{\sigma}^{i} q(\sigma) \cdot z^{i}(\sigma) \geq 0 & \forall s \in S .
\end{array}\right\}
$$


Since $\bar{x}^{i}$ is optimal in the budget set $B_{M Q}^{i}\left(\bar{p}, q, \bar{\lambda}^{i}\right)$, it follows that $U^{i} \cap A^{i}=\emptyset$; moreover the assumption $C 4$ implies that $\bar{x}^{i}+1 \in \operatorname{int}\left(U^{i}\right)$. As previously, we can apply the Hahn-Banach theorem to get the existence of some non-zero element $P^{i}$ in $\left(\ell_{\infty}(S \times\right.$ $L))_{+}^{\prime}=(b a(S \times L))_{+}$such that for all $\left(x^{i}, y^{i}\right) \in A^{i} \times U^{i}$, one has $P^{i} \cdot x^{i} \leq P^{i} \cdot y^{i}$. Since $\bar{x}^{i} \in B_{M Q}^{i}\left(\bar{p}, q, \bar{\lambda}^{i}\right) \subset A^{i}$, and since by local non-satiation $\bar{x}^{i} \in \operatorname{cl}\left(U^{i}\right)$, one deduces that for all $\left(x^{i}, y^{i}\right) \in A^{i} \times U^{i}$, one has $P^{i} \cdot x^{i} \leq P^{i} \cdot \bar{x}^{i} \leq P^{i} \cdot y^{i}$. Since $P^{i}$ is in $b a(S \times L)_{+}$, we can apply the Yosida-Hewitt theorem to get the existence of some $\tilde{\pi}^{i} \in \ell_{1}^{+}(S \times L)$ such that $P^{i}$ and $\pi^{i}$ coincide on "finite" elements of $\ell_{\infty}(S \times L)$.

As previously, we prove that $\bar{x}^{i}$ is optimal for agent $i$ in the set $\left\{x^{i} \in X^{i} \mid \tilde{\pi}^{i} \cdot x^{i} \leq \tilde{\pi}^{i} \cdot \bar{x}^{i}\right\}$. In particular, we deduce from Assumption $C 4$ that $\tilde{\pi}^{i}(s)>0$, for every node $s$.

Let us now construct the node prices $\tilde{\lambda}^{i}$ corresponding to the present value prices $\tilde{\pi}^{i}$. For any time horizon $T$, let us consider the set $B_{T}^{i}(\bar{p}, q)$ defined as in the proof of Theorem 3. Since $B_{T}^{i}(\bar{p}, q)$ is clearly a subset of $A^{i}$, it follows that $\tilde{\pi}^{i} \cdot x^{i} \chi_{S^{T}} \leq \tilde{\pi}^{i} \cdot \bar{x}^{i}$ for all $x^{i} \in B_{T}^{i}(\bar{p}, q)$. We have proved that the obviously consistent system of inequalities defining $B_{T}^{i}(\bar{p}, q)$ implies that $\sum_{s \in S^{T}} \tilde{\pi}^{i}(s) \cdot x^{i}(s) \leq \tilde{\pi}^{i} \cdot \bar{x}^{i}$. As previously, there exists $\left(\left(\tilde{\lambda}_{s}^{i, T}\right)_{s \in S^{T}},\left(\mu_{s}^{i, T}\right)_{s \in S_{T+1}}\right) \in \mathbb{R}_{+}^{S^{T}} \times \mathbb{R}_{+}^{S_{T+1}}$ such that :

$$
\begin{aligned}
& \tilde{\pi}^{i}(s)=\tilde{\lambda}_{s}^{i, T} \bar{p}(s) \text { for all } s \text { in } S^{T} \\
& \tilde{\lambda}_{s}^{i, T} q(s)=\sum_{\sigma \in s^{+}} \tilde{\lambda}_{\sigma}^{i, T} r(\sigma) \text { for all } s \text { in } S^{T-1} \\
& \tilde{\lambda}_{s}^{i, T} q(s)=\sum_{\sigma \in s^{+}} \mu_{\sigma}^{i, T} r(\sigma) \text { for all } s \text { in } S_{T} ; \\
& \text { and } \tilde{\pi}^{i} \cdot \bar{x}^{i} \geq \sum_{s \in S^{T}} \tilde{\lambda}_{s}^{i, T} \bar{p}(s) \cdot \omega^{i}(s) .
\end{aligned}
$$

Recalling that $\tilde{\pi}^{i}(s)>0$ for all $s \in S$, we deduce from (3) that the real number $\tilde{\lambda}_{s}^{i, T}$ is positive and independent of $T$. This allow the construction of some $\tilde{\lambda}^{i}$ in $\mathbb{R}_{++}^{S}$ satisfying $\tilde{\pi}^{i}(s)=\tilde{\lambda}_{s}^{i} \bar{p}(s)$ for all $s$ in $S$; moreover using (4), one also has $\tilde{\lambda}_{s}^{i} q(s)=\sum_{\sigma \in s^{+}} \tilde{\lambda}_{\sigma}^{i} r(\sigma)$ for all $s$ in $S$. Since $\tilde{\pi}^{i} \cdot \omega^{i} \leq \tilde{\pi}^{i} \cdot \bar{x}^{i}$, one deduces that $B^{i}\left(\bar{p}, q, \tilde{\lambda}^{i}\right) \subset\left\{x^{i} \in X^{i} \mid \tilde{\pi}^{i} \cdot x^{i} \leq \tilde{\pi}^{i} \cdot \omega^{i}\right\} \subset$ $\left\{x^{i} \in X^{i} \mid \tilde{\pi}^{i} \cdot x^{i} \leq \tilde{\pi}^{i} \cdot \bar{x}^{i}\right\}$. Recalling that $\bar{x}^{i}$ is optimal in the last set, it suffices to prove that $\left(\bar{x}^{i}, \bar{z}^{i}\right) \in B^{i}\left(\bar{p}, q, \tilde{\lambda}^{i}\right)$ to get the optimality of $\left(\bar{x}^{i}, \bar{z}^{i}\right)$ in $B^{i}\left(\bar{p}, q, \tilde{\lambda}^{i}\right)$.

To this end, let us consider a node $s$ and an agent $i$. Let $\left(\tilde{x}_{s}^{i}, \tilde{z}_{s}^{i}\right)$ be the consumption/portfolio plan defined by $\left(\bar{x}^{i} \chi_{S \backslash S^{+}(s)}+1_{s}+\beta \bar{x}^{i} \chi_{S^{+}(s)}, \bar{z}^{i} \chi_{S \backslash S(s)}+\beta \bar{z}^{i} \chi_{S(s)}\right)$. Since by assumption $C 5, \tilde{x}_{s}^{i} \succ^{i} \bar{x}^{i}$, one deduces that $\left(\tilde{x}_{s}^{i}, \tilde{z}_{s}^{i}\right) \notin B_{M Q}^{i}\left(\bar{p}, q, \bar{\lambda}^{i}\right)$. Since $\left(\tilde{x}_{s}^{i}, \tilde{z}_{s}^{i}\right)$ obviously satisfies the Magill-Quinzii transversality condition in every node and the spot constraint in every node $s^{\prime} \neq s$, it follows that the spot constraint in node $s$ is not satisfied. 
As previously, one gets

$$
\sum_{\substack{s \in S_{T} \\ q(s) \cdot \bar{z}^{i}(s) \leq 0}} \tilde{\lambda}_{s}^{i} q(s) \cdot \bar{z}^{i}(s)>-\frac{m-1}{1-\beta} \sum_{\substack{s \in S_{T} \\ q(s) \cdot \bar{z}^{i}(s) \leq 0}} \tilde{\lambda}_{s}^{i}\|\bar{p}(s)\|_{1} .
$$

Hence we deduce that

$$
0 \geq \sum_{\substack{s \in S_{T} \\ q(s) \cdot \bar{z}^{i}(s) \leq 0}} \tilde{\lambda}_{s}^{i} q(s) \cdot \bar{z}^{i}(s)>-\frac{m-1}{1-\beta} \sum_{s \in S_{T}} \tilde{\lambda}_{s}^{i}\|\bar{p}(s)\|_{1}=-\frac{m-1}{1-\beta} \tilde{\pi}^{i} \cdot 1 \chi s_{T} .
$$

Since $\tilde{\pi}^{i}$ is in $\ell_{1}(S \times L)$, the right term tends to zero, when $T$ goes to infinity. Hence one has $\left(\bar{x}^{i}, \bar{z}^{i}\right) \in B^{i}\left(\bar{p}, q, \tilde{\lambda}^{i}\right)$.

Remark 8. We emphasize that in Theorem 5, the "initial" present value price $\pi^{i} \stackrel{\text { def }}{=}$ $\left(\bar{\lambda}_{s}^{i} \bar{p}(s)\right)_{s \in S}$ does not necessarily belong to $\ell_{1}(S \times L)$.

Remark 8 allows us to summarize Theorems 2, 3,4 and 5. Actually, we state an obvious equivalence result.

Theorem 6. Assume that the economy $\mathcal{E}=\left(\left(X^{i}, \succsim^{i}, \omega^{i}\right)_{i=1}^{m}, S, R\right)$ satisfies Assumptions $C 1-C 5$ and $F$; then for any consumption/portfolio plan $\left(\bar{x}^{i}, \bar{z}^{i}\right)_{i=1}^{m}$, and for any price $\bar{p}$, the following assertions are equivalent:

(i) There exists some system of debt constraints $\bar{D}^{i}$ such that $\left(\left(\bar{x}^{i}, \bar{z}^{i}, \bar{D}^{i}\right)_{i=1}^{m}, \bar{p}, q\right)$ is an equilibrium with debt constraints of $\mathcal{E}$.

(ii) $\left(\left(\bar{x}^{i}, \bar{z}^{i}\right)_{i=1}^{m}, \bar{p}, q\right)$ is a finitely effective equilibrium of the economy $\mathcal{E}$.

(iii) $\left(\left(\bar{x}^{i}, \bar{z}^{i}\right)_{i=1}^{m}, \bar{p}, q\right)$ is a bounded debt equilibrium of $\mathcal{E}$.

(iv) $\left(\left(\bar{x}^{i}, \bar{z}^{i}\right)_{i=1}^{m}, \bar{p}, q\right)$ is an equilibrium with an implicit borrowing limit

(v) There exists some present value node prices $\bar{\lambda}^{i}$ such that $\left(\left(\bar{x}^{i}, \bar{z}^{i}, \bar{\lambda}^{i}\right)_{i=1}^{m}, \bar{p}, q\right)$ is an equilibrium with present value node prices of $\mathcal{E}$.

(vi) There exists some present value node prices $\tilde{\lambda}^{i}$. such that $\left(\left(\bar{x}^{i}, \bar{z}^{i}, \tilde{\lambda}^{i}\right)_{i=1}^{m}, \bar{p}, q\right)$ is a $M Q$-equilibrium with present value node prices of $\mathcal{E}$.

The equivalence between ( $i),($ ii), (iii) was proved by Levine and Zame (1992); Magill and Quinzii (1994) establish the equivalence between ( $i v)$ and (vi). 


\section{Appendix}

\section{Proof of Lemma 2.}

It follows from the closedness of $\operatorname{Im} \Phi$, that $\Gamma$ is closed. If $\Gamma$ is unbounded, there exists some non-zero vector $\bar{\varphi}$ in the asymptotic cone of $\Gamma$. Since this set is bounded below, one has $\bar{\varphi} \geq 0$. Moreover, $\bar{\varphi}$ is also in the asymptotic cone of $\operatorname{Im} \Phi_{\text {s }}$ which is a linear space. Finally, we have proved the existence of an element $\bar{\varphi}>0$ in the set $\operatorname{Im} \Phi_{\boldsymbol{s}}$, which contradicts the no-arbitrage condition.

\section{Proof of Proposition 2.}

To prove Proposition 2, we will first state a lemma.

Lemma 4. Let $D$ be a system of debt constraints. If $q$ is arbitrage-free,

(i) If $D$ is not $(p, q)$-consistent, there exists some system of debt constraints $D^{\prime}$ such that $D^{\prime}>D$ and $B^{i}\left(p, q, D^{\prime}\right)=B^{i}(p, q, D)$.

(ii) If $D$ is $(p, q)$-loose and not $(p, q)$-consistent, there exists some system of debt constraints $D^{\prime}$ such that $D^{\prime}>D, B^{i}\left(p, q, D^{\prime}\right)=B^{i}(p, q, D)$ and $D^{\prime} i s(p, q)$-loose.

\section{Proof.}

(i) Since $D$ is not $(p, q)$-consistent, let $s$ be a node such that $D$ is not $(p, q)$-consistent at node $s$. Hence, for all portfolio $z(s) \in \mathbb{R}^{J_{e}}$, one has

$r(\sigma) \cdot z(s) \geq D(\sigma)$ for all $\sigma \in s^{+} \Rightarrow D(s)+p(s) \cdot \omega^{i}(s)-q(s) \cdot z(s)<0$.

Let us define $D^{\prime}(s)=\inf \Delta$ where $\Delta$ is the set

$$
\left\{\begin{array}{l|ll}
d \in \mathbb{R}^{-} & \exists z(s) \in \mathbb{R}^{J_{e}} \begin{array}{l}
d+p(s) \cdot \omega^{i}(s)-q(s) \cdot z(s) \geq 0 \\
r(\sigma) \cdot z(s) \geq D(\sigma) \text { for all } \sigma \in s^{+}
\end{array}
\end{array}\right\}
$$

First, we remark that this set is non-empty since we can take $d=0$ associated with $z(s)=0$. It follows that if $D^{\prime}(s)$ exists, then $D^{\prime}(s) \leq 0$. An equivalent formulation for $\Delta$ is $\Delta=\left\{d \in \mathbb{R}^{-} \mid \Gamma(d) \neq \emptyset\right\}$ with

$$
\Gamma(d)=\left\{\varphi \in I_{m} \Phi_{s} \mid \varphi \geq\left(\begin{array}{c}
-d-p(s) \cdot \omega^{i}(s) \\
(D(\sigma))_{\sigma \in s^{+}}
\end{array}\right)\right\} \subset\left\{\varphi \in I_{m} \Phi_{s} \mid \varphi \geq\left(\begin{array}{c}
-p(s) \cdot \omega^{i}(s) \\
(D(\sigma))_{\sigma \in s^{+}}
\end{array}\right)\right\} .
$$

The second set is then non-empty and it follows from Lemma 2 that it is compact. Hence we deduce that $\Delta$ is bounded below, and this proves the existence of $D^{\prime}(s)$. Moreover, $\Delta$ is closed. Indeed, let $d^{\nu} \rightarrow d$; for every $\nu$, there exists $\varphi^{\nu} \in I_{m} \Phi_{s}$ and $\varphi^{\nu} \geq$ 
$\left(\begin{array}{c}-d^{\nu}-p(s) \cdot \omega^{i}(s) \\ (D(\sigma))_{\sigma \in s^{+}}\end{array}\right)$. With no loss of generality, one can assume $\varphi^{\nu} \rightarrow \varphi \in \operatorname{Im} \Phi_{s}$ and $\varphi \geq\left(\begin{array}{c}-d-p(s) \cdot \omega^{i}(s) \\ (D(\sigma))_{\sigma \in s^{+}}\end{array}\right)$, so that $d \in \Delta$.

Finally $D^{\prime}(s)=\min \Delta$ and for some $z(s) \in \mathbb{R}^{J_{0}}$, consequently one has

$$
D^{\prime}(s)+p(s) \cdot \omega^{i}(s)-q(s) \cdot z(s) \geq 0>D(s)+p(s) \cdot \omega^{i}(s)-q(s) \cdot z(s)
$$

which shows that $D^{\prime}(s)>D(s)$. We can now define $D^{\prime}\left(s^{\prime}\right)=D\left(s^{\prime}\right)$ for all $s^{\prime} \neq s$. By definition of $D^{\prime}, D^{\prime}$ is a system of debt constraints satisfying $D<D^{\prime}$.

Thus it only remains to show that the two systems of debt constraints give the same budget set. First we remark that it follows from the inequality between $D$ and $D^{\prime}$ that $B^{i}\left(p, q, D^{\prime}\right) \subset B^{i}(p, q, D)$. Conversely, let us take any consumption plan $x$ in the budget set $B^{i}(p, q, D)$ sustained by the portfolio plan $z$. We want to prove that for each node $s^{\prime}$, one has $r\left(s^{\prime}\right) \cdot z\left(s^{\prime-}\right) \geq D^{\prime}\left(s^{\prime}\right)$. Since $D$ and $D^{\prime}$ differ in node $s$ only, it suffices to prove that $r(s) \cdot z\left(s^{-}\right) \geq D^{\prime}(s)$. But it follows from the budget/debt constraints that

$$
\left\{\begin{array}{l}
r(s) \cdot z\left(s^{-}\right)+p(s) \cdot\left(\omega^{i}(s)-x(s)\right)-q(s) \cdot z(s) \geq 0, \\
\text { for all } \sigma \in s^{+}, r(\sigma) \cdot z(s) \geq D(\sigma) .
\end{array}\right.
$$

One deduces that

$$
\left\{\begin{array}{l}
r(s) \cdot z\left(s^{-}\right)+p(s) \cdot \omega^{i}(s)-q(s) \cdot z(s) \geq 0, \\
\text { for all } \sigma \in s^{+}, r(\sigma) \cdot z(s) \geq D(\sigma)
\end{array}\right.
$$

Hence $r(s) \cdot z\left(s^{-}\right) \in \Delta$, and by definition of $D^{\prime}(s)$, one obtains $r(s) \cdot z\left(s^{-}\right) \geq D^{\prime}(s)$.

(ii) Let us define $D^{\prime}$ as above. Clearly, one has $D^{\prime}>D$ and $B^{i}\left(p, q, D^{\prime}\right)=B^{i}(p, q, D)$. Since $D$ and $D^{\prime}$ differ on node $s$ only, $D^{\prime}$ is also $(p, q)$-loose in each node of $D \backslash\left\{s, s^{-}\right\}$. By construction, $D^{\prime}$ is $(p, q)$-loose at $s$. If $s \neq 0$, let us take $z\left(s^{-}\right) \in \mathbb{R}^{J_{0}-\text { satisfying }}$ $r(\sigma) \cdot z\left(s^{-}\right) \geq D^{\prime}(\sigma)$ for all $\sigma \in\left(s^{-}\right)^{+}$. Since $D^{\prime}>D$, one deduces that $r(\sigma) \cdot z\left(s^{-}\right) \geq D(\sigma)$ for all $\sigma \in\left(s^{-}\right)^{+}$. This assertion together with the $(p, q)$-looseness of $D$ at node $s^{-}$leads to $D\left(s^{-}\right)+p\left(s^{-}\right) \cdot \omega^{i}\left(s^{-}\right)-q\left(s^{-}\right) \cdot z\left(s^{-}\right) \leq 0$. Since $D$ and $D^{\prime}$ coincide at node $s^{-}, D^{\prime}$ is also $(p, q)$-loose at node $s^{-}$and consequently $D^{\prime}$ is $(p, q)$-loose.

(a) Proof of Assertion (i) of Proposition 2.

Recall that the initial system of debt constraints is denoted by $D^{i}$. 
Let $X$ be the set of systems of debt constraints defined by $X=\left\{D \mid B^{i}(p, q, D)=\right.$ $B^{i}\left(p, q, D^{i}\right)$ and $\left.D \geq D^{i}\right\}$. We will consider the classical ordering on $X, D \leq D^{\prime}$ if and only if for all $s$ in $S$, one has $D(s) \leq D^{\prime}(s)$. We now claim that every totally ordered subset of $X$ has an upper bound.

Indeed, let us consider a totally ordered subset of $X,\left(D^{\theta}\right)_{\theta \in \Theta}$. Since for every node $s$, for all $\theta, D^{\theta}(s) \leq 0$, we can define $\bar{D}(s)$ by $\bar{D}(s)=\sup \left\{D^{\theta}(s) \mid \theta \in \Theta\right\}$. Clearly $\bar{D}$ is a system of debt constraints satisfying for all $\theta, \bar{D} \geq D^{\theta} \geq D^{i}$. Consequently, one obtains $B^{i}(p, q, \bar{D}) \subset B^{i}\left(p, q, D^{i}\right)$. Conversely, let us take any consumption plan $x$ in the budget set $B^{i}\left(p, q, D^{i}\right)$ sustained by the portfolio plan $z$. We want to prove that $r(s) \cdot z\left(s^{-}\right) \geq \bar{D}(s)$ for every node $s$. Since for all $\theta$, one has $B^{i}\left(p, q, D^{\theta}\right)=B^{i}\left(p, q, D^{i}\right)$, one obtains that $r(s) \cdot z\left(s^{-}\right) \geq D^{\theta}(s)$ for every node $s$. By taking the supremum in $\theta \in \Theta$, one gets the required inequality. Hence, $\bar{D} \in X$, and the set $\left(D^{\theta}\right)_{\theta \in \Theta}$ has an upper bound.

We can apply Zorn's lemma to get the existence of a maximal element $\tilde{D}^{i}$ in the set $X$. To end the proof, since $\tilde{D}^{i}$ is in $X$, it remains to prove that $\tilde{D}^{i}$ is $(p, q)$-consistent. Indeed, if $\tilde{D}^{i}$, is not $(p, q)$-consistent, we can apply lemma 4 to get the existence of a system of debt constraints $D^{\prime}$ in $X$ such that $D^{\prime}>\tilde{D}^{i}$. This contradicts the definition of $\tilde{D}^{i}$.

\section{(b) Proof of Assertion (ii)}

The proof of Assertion (ii) is similar to the one of Assertion (i). We replace the set X by the set $X^{\prime}=\left\{D\right.$ such that $B^{i}(p, q, D)=B^{i}\left(p, q, D^{i}\right), D \geq D^{i}$ and $\mathrm{D}$ is $(p, q)$-loose $\}$. As before, one checks that every totally ordered subset of $X^{\prime}$ has an upper bound and that a maximal element of $X^{\prime}$ satisfies the condition of Assertion (ii).

\section{Proof of Proposition 3.}

Let us recall the definition of $\bar{D}^{i, T}$;

$$
\bar{D}^{i, T}(s)=\inf \left\{\bar{p}^{T}(s) \cdot\left(x^{i}(s)-\omega^{i}(s)\right)+q(s) \cdot z^{i}(s)\right\}
$$

where the infimum is taken among all $\left(x^{i}, z^{i}\right)$ belonging to $B^{i, T}\left(\bar{p}^{T}, q\right)$. Note that $\forall s \in S_{T}$, $\bar{D}^{i, T}(s)=-\bar{p}^{T}(s) \cdot \omega^{i}(s)$ and $\forall s \notin S^{T}, \bar{D}^{i, T}(s)=0$. Exactly as in the proof of Proposition 2 , one deduces from the no-arbitrage condition on $q$ that $\bar{D}^{i, T}(s)$ is actually a minimum. Then it is obvious that $\bar{D}^{i, T}$ is $\left(\bar{p}^{T}, q\right)$-loose and consistent.

The inequality $\bar{D}^{i, T}(s) \leq D_{f}^{i}\left(s, \bar{p}^{T}, q\right)$ follows from $\left(\bar{p}^{T}, q\right)$-looseness. On the other hand, it follows from the definition of $\bar{D}^{i, T}$ that $\forall s \in S^{T}, \bar{D}^{i, T}(s) \geq D_{f}^{i}\left(s, \bar{p}^{T}, q\right)$, which ends the proof. 


\section{Proof of Proposition 4.}

We first introduce some notations. We will denote by $\Omega$ the set $[0,+\infty[\times[0,1] \times]-\infty, 0[$ and we will consider the real valued function $\phi: \Omega \rightarrow[0,1]$ defined by $\phi(\alpha, \beta, \gamma)=$ $\begin{cases}\frac{\alpha+\beta \gamma}{\alpha+\gamma} & \text { if } \alpha+\beta \gamma<0 \\ 0 & \text { if } \alpha+\beta \gamma \geq 0\end{cases}$

We will construct a process $\left(\beta^{i}(s)\right)_{s \in S\left(s_{0}\right)}$ by induction on $s$. $\left\{\begin{array}{l}\beta^{i}\left(s_{0}^{-}\right)=d / D^{i}\left(s_{0}\right) \\ \forall s \in S\left(s_{0}\right), \beta^{i}(s)=\phi\left(\bar{p}(s) \cdot \omega^{i}(s), \beta^{i}\left(s^{-}\right), D^{i}(s)\right)\end{array}\right.$

We will prove that $\beta^{i}(s)$ is equal to zero except for a finite number of node $s$. Notice that $\beta^{i}(s)=0$ implies that $\beta^{i}(\sigma)=0$ for all node $\sigma \in s^{+}$. Since for all $(\alpha, \beta, \gamma) \in \Omega$, $\phi(\alpha, \beta, \gamma) \leq \beta$, one deduces that $\beta^{i}(s) \leq \beta^{i}\left(s^{-}\right)$for all node $s \in S\left(s_{0}\right)$.

Let us now consider a node $s$ such that $\beta^{i}(s)>0$. Since on $\Omega, \phi$ is a decreasing function of $\gamma$ and recalling that our assumption gives the existence of some real number $\Delta(\Delta \leq 0)$ independant of $s$ satisfying $D^{i}(s) \geq \Delta\|\bar{p}(s)\|_{1}$, one deduces that $\beta^{i}(s) \leq$ $\phi\left(\bar{p}(s) \cdot \omega^{i}(s), \beta^{i}\left(s^{-}\right), \Delta\|\bar{p}(s)\|_{1}\right)$.

Moreover since $\omega^{i} \in \operatorname{int}\left(\ell_{\infty}^{+}(S \times L)\right)$, there exists some real number $m^{i}$ independant of $s$ satisfying $\bar{p}(s) \cdot \omega^{i}(s) \geq m^{i}\|\bar{p}(s)\|_{1}$. Since on $\Omega, \phi$ is a decreasing function of $\alpha$, one deduces that

$$
\beta^{i}(s) \leq \phi\left(m^{i}\|\bar{p}(s)\|_{1}, \beta^{i}\left(s^{-}\right), \Delta\|\bar{p}(s)\|_{1}\right)=\phi\left(m^{i}, \beta^{i}\left(s^{-}\right), \Delta\right)=\frac{m^{i}+\beta^{i}\left(s^{-}\right) \Delta}{m^{i}+\Delta} .
$$

Since $\beta^{i}\left(s^{-}\right) \leq \beta^{i}\left(s_{0}\right)$, one deduce that

$$
\beta^{i}(s) \leq \beta^{i}\left(s^{-}\right)+\frac{m^{i}\left(1-\beta^{i}\left(s_{0}\right)\right)}{m^{i}+\Delta} \text { for all node } s \text { such that } \beta^{i}(s)>0 .
$$

Since $\frac{m^{i}\left(1-\beta^{i}\left(s_{0}\right)\right)}{m^{i}+\Delta}<0$, there exists a time period $T$ such that for all node $s \in S\left(s_{0}\right)$, one has $\tau(s) \geq \tau\left(s_{0}\right)+T \Rightarrow \beta^{i}(s)=0$.

We recall that the consistency of $D^{i}$ at node $s$ implies the existence of some portfolio $z^{i}(s) \in \mathbb{R}^{J_{0}}$ satisfying $\left\{\begin{array}{l}D^{i}(s)+p(s) \cdot \omega^{i}(s)-q(s) \cdot z^{i}(s) \geq 0, \\ \text { and for all } \sigma \in s^{+}, r(\sigma) \cdot z^{i}(s) \geq D^{i}(\sigma) .\end{array}\right.$

We can now construct the portfolio $\left(\bar{z}^{i}(s)\right)_{s \in S\left(s_{0}\right)}$ by $\bar{z}^{i}(s)=\beta^{i}(s) z^{i}(s)$. It follows from the definition of $\left(\bar{z}^{i}(s)\right)_{s \in S\left(s_{0}\right)}$ that it repays the debt $d$ in finite time from node $s_{0}$. 


\section{References}

Bewley, T. (1972), Existence of equilibria in economies with infinitely many commodities, Journal of Economic Theory, 49, 514-540.

Brown, D. and L. Lewis (1981), Myopic economic agents, Econometrica, 49: 359-368.

Florenzano, M. and P. Gourdel (1994), T-period economies with incomplete markets, Economics Let ters, 44, 91-97.

Hernandez, A. and M. Santos (1991), Incomplete financial markets in infinite horizon economies, Working paper, University of Wisconsin. Revised version (1992), under the new title : Competitive equilibria for infinitehorizon economies with incomplete financial markets.

Hernandez, A. and M. Santos (1994), Competitive equilibria for infinite-horizon economies with incomplete markets, Working paper, ITAM, Mexico.

Levine, D. (1989), Infinite horizon economies with incomplete markets, Journal of Mathematical Economics, 18: 357-376.

Levine, D. and W. Zame (1992), Debt constraints and equilibrium in infinite horizon economies with incomplete markets, Discussion paper, University of California, Los Angeles. (Forthcoming in Journal of Mathematical Economics.)

Magill, M. and M. Quinzii (1994), Infinite Horizon Incomplete Markets, Econometrica, Volume 62-4, 853-880.

Mas-Colell, A. and W. Zame (1991), Equilibrium theory in infinite dimensional spaces, Handbook of Mathematical Economics, Volume IV, North Holland, Amsterdam.

Rockafellar, R.T. (1970), Convex Analysis, Princeton University Press, Princeton.

Santos, M. and M. Woodford (1993), Rational asset pricing bubbles, Working paper, Universidad Carlos III de Madrid.

Schmachtenberg, R. (1988), Stochastic overlapping generations model with incomplete markets, Working paper, University of Mannheim. 